\title{
Two new Leptobrachella species (Anura, Megophryidae) from the Yunnan-Guizhou Plateau, southwestern China
}

\author{
Jian Wang ${ }^{1,2}$, Zhi-Tong Lyu', Shuo Qi', Zhao-Chi Zeng ${ }^{1,2}$, \\ Wen-Xiang Zhang', Long-Shan Lu', Ying-Yong Wang'
}

I State Key Laboratory of Biocontrol / The Museum of Biology, School of Life Sciences, Sun Yat-sen University, Guangzhou 510275, China 2 Shenzhen Shuanghuamu Biological Technology Co., Ltd, Shenzhen 51800, China 3 Yunnan Huanglianshan National Nature Reserve, Honghe Hani and Yi Autonomous Prefecture, Yunnan Province 662500, China

Corresponding author: Ying-Yong Wang (wangyy@mail.sysu.edu.cn)

Academic editor: A. Ohler | Received 28 June 2020 | Accepted 26 October 2020 | Published 18 November 2020

http://zoobank.org/E5F4F51A-99CF-405F-BD0C-06C8DF271410

Citation: Wang J, Lyu Z-T, Qi S, Zeng Z-C, Zhang W-X, Lu L-S, Wang Y-Y(2020) Two new Leptobrachella species (Anura, Megophryidae) from the Yunnan-Guizhou Plateau, southwestern China. ZooKeys 995: 97-125. https://doi. org/10.3897/zookeys.955.55939

\begin{abstract}
Two new toad species of the genus Leptobrachella are described from the Yunnan-Guizhou Plateau of China, based on the combination of molecular and morphological data. The description of Leptobrachella aspera Wang, Lyu, Qi \& Wang, sp. nov. from Huanglianshan Nature Reserve represents the thirteenth Leptobrachella species known from Yunnan Province, and the description of Leptobrachella dorsospina Wang, Lyu, Qi \& Wang, sp. nov. from Yushe Forest Park represents the sixth Leptobrachella species known from Guizhou Province. These new discoveries further emphasize the extremely high diversity of the Leptobrachella toads in these regions.
\end{abstract}

\section{Keywords}

Leptobrachella aspera sp. nov., Leptobrachella dorsospina sp. nov., molecular phylogeny, morphology, taxonomy

Copyright Jian Wang et al. This is an open access article distributed under the terms of the Creative Commons Attribution License (CC BY 4.0), which permits unrestricted use, distribution, and reproduction in any medium, provided the original author and source are credited. 


\section{Introduction}

The generic classifications within the family Megophryidae Bonaparte, 1850 have always been controversial. For example, recent comprehensive approaches have produced different taxonomic schemes for the genus Megophrys sensu lato Kuhl and Van Hasselt 1822 (Chen et al. 2017; Mahony et al. 2017; Liu et al. 2018; Li et al. 2020b). The taxonomy of another group of megophrid toads are facing the same problem: Chen et al. (2018) presented the first well-resolved phylogenetic hypothesis for the genera Leptolalax Dubois, 1983 and Leptobrachella Bonaparte, 1850. They tended towards the most conservative "one-genus option" pending the acquisition of additional data by assigning Leptolalax as a junior synonym of Leptobrachella. Their results also rejected the hypothesis that Leptolalax consists of two subgenera as proposed by Delorme et al. (2006) and Dubois et al. (2010). In this context, the genus Leptobrachella currently contains 82 species widely distributed from southern China, west to northeastern India, through Indochina to the island of Borneo (Frost 2020). Leptobrachella is a species-rich genus of megophrid frogs, and a large number of new species have been discovered in recent years due to the application of integrative taxonomy incorporating detailed morphological, bioacoustic and molecular analyses (Rowley et al. 2016, 2017; Yang et al. 2016; Yuan et al. 2017; Eto et al. 2018; Nguyen et al. 2018; Wang et al. 2019; Chen et al. 2020; Luo et al. 2020; Qian et al. 2020).

During recent field surveys in the Yunnan-Guizhou Plateau of southwestern China, a number of megophrid specimens were collected from Yushe Forest Park in western Guizhou (Fig. 1, site 1) and Huanglianshan Nature Reserve in southern Yunnan (Fig. 1, site 2), respectively. Morphologically, all the specimens can be assigned to the genus "Leptolalax" (now a junior subjective synonym of Leptobrachella), based on the following characters: (1) small or moderate size, snout-vent length not greater than $60.0 \mathrm{~mm}$, (2) rounded finger tips, the presence of an elevated inner palmar tubercle not continuous to the thumb, (3) presence of macroglands on body including supra-axillary, pectoral, femoral and ventrolateral glands, (4) vomerine teeth absent, (5) tubercles on eyelids present, and (6) anterior tip of snout with whitish vertical bar (Dubois 1983; Matsui 1997, 2006; Lathrop et al. 1998; Delorme et al. 2006; Das et al. 2010). Although their generic allocation is without doubt, some characters of these specimens do not correspond to the diagnoses of any recognized species. Subsequent molecular analysis further revealed that these specimens represent two distinct evolutionary lineages. Considering both the morphological differences and molecular divergences, these specimens are described herein as two new species.

\section{Materials and methods}

\section{Sampling}

For the molecular analyses, a total of 80 sequences (nine muscle tissue samples was sequenced and 71 sequences obtained from GenBank) were used, including five sequences of the undescribed species from Guizhou, four sequences of the undescribed species 


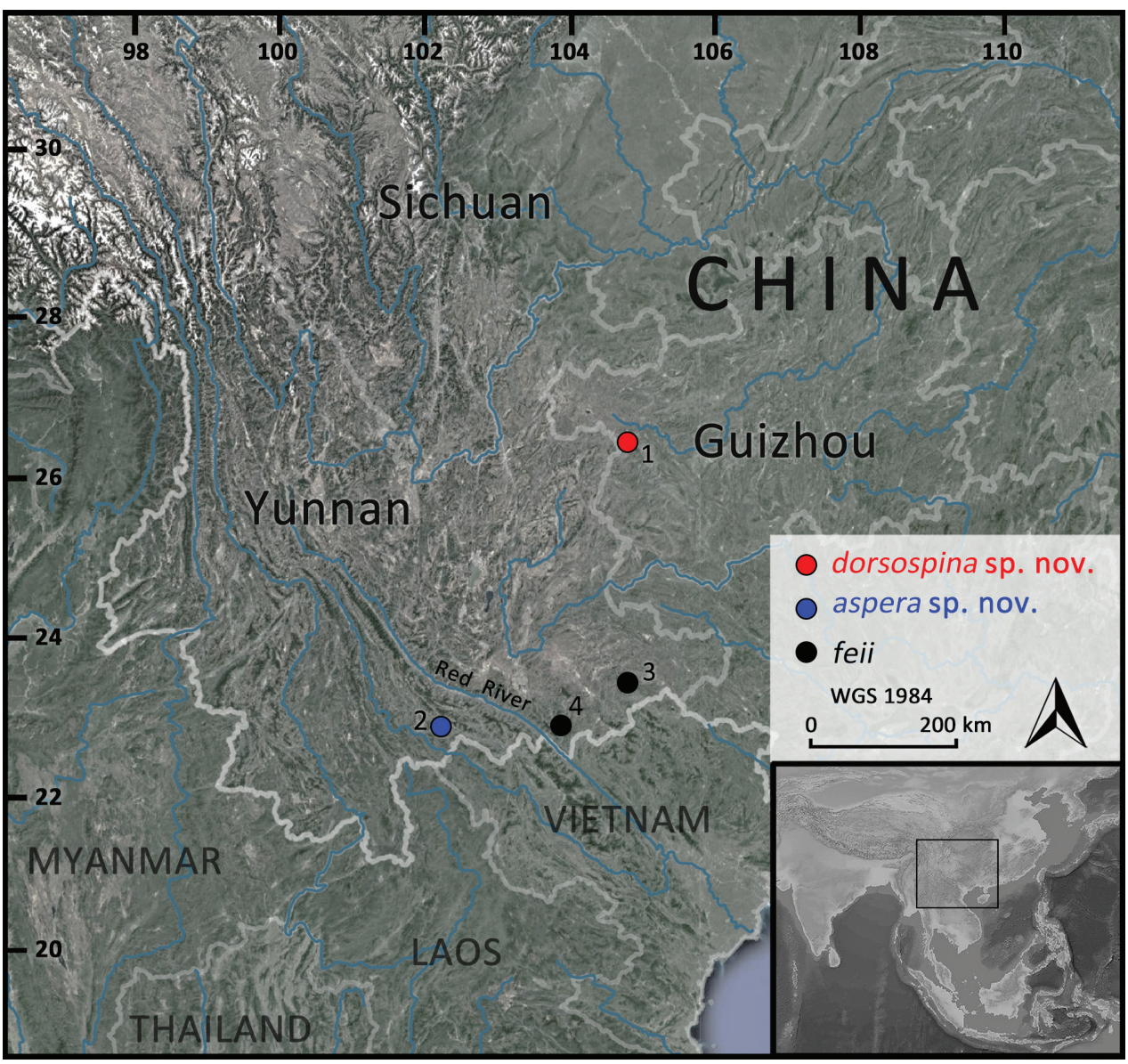

Figure I. Collection sites. Site 1-Yushe Forest Park, Shuicheng County, Guizhou Province, the type locality of Leptobrachella dorsospina sp. nov.; site 2-Huanglianshan Nature Reserve, Lyuchun County, Yunnan Province, the type locality of L. aspera sp. nov.; site 3-Xiaoqiaogou Nature Reserve, Xichou County, Yunnan Province, the type locality of L. feii; and site 4-Daweishan Nature Reserve, Pingbian County, Yunnan Province, another distribution locality of L. feii.

from Yunnan, 69 sequences of 66 recognized congeners, and two out-group sequences of Oreolalax rhodostigmatus $\mathrm{Hu} \&$ Fei, 1979 and Leptobrachium tengchongensis Yang \& Huang, 2019, respectively (Table 1). Due to the presence of cryptic diversity within genus Leptobrachella, we chose sequences from type series or topotype specimens for molecular analysis if available to ensure the taxonomic identity of the species being studied.

\section{DNA Extraction, PCR, and sequencing}

DNA was extracted from muscle tissue using a DNA extraction kit from Tiangen Biotech (Beijing) Co., Ltd. The mitochondrial gene $16 \mathrm{~S}$ ribosomal RNA gene (16S rRNA) fragment from each sample was sequenced. Fragments were amplified using the primer 
pairs L3975 (5'-CGCCTGTTTACCAAAAACAT-3') and H4551 (5'-CCGGTCTGAACTCAGATCACGT-3') (Simon et al. 1994). PCR amplifications were performed in a $20 \mu \mathrm{l}$ reaction volume with the following cycling conditions: an initial denaturing step at $95^{\circ} \mathrm{C}$ for five min; 35 cycles of denaturing at $95^{\circ} \mathrm{C}$ for $40 \mathrm{~s}$, annealing at $53{ }^{\circ} \mathrm{C}$ for $40 \mathrm{~s}$ and extending at $72{ }^{\circ} \mathrm{C}$ for one min; and a final extending step of $72{ }^{\circ} \mathrm{C}$ for $10 \mathrm{~min}$. PCR products were purified with spin columns. The purified products were sequenced with both forward and reverse primers using BigDye Terminator Cycle Sequencing Kit according to the guidelines of the manufacturer. The products were sequenced on an ABI Prism 3730 automated DNA sequencer in Shanghai Majorbio Biopharm Technology Co., Ltd. All sequences have been deposited in GenBank (Table 1).

\section{Phylogenetic analyses}

Sequences were aligned in Clustal X 2.0 (Thompson et al. 1997) with default parameters. For GenBank sequences which lack information for part of the missing segments, we filled the blank sites with "N". The aligned data was trimmed allowing no gap positions and default parameters in Gblocks version 0.91b (Castresana 2000). lyWe ran Jmodeltest v2.1.2 (Darriba et al. 2012) with Akaike and Bayesian information criteria on the alignment and obtained the best-fitting nucleotide substitution model of GTR $+\mathrm{I}+\mathrm{G}$. Phylogenetic analysis was using Bayesian inference (BI) in MrBayes 3.2.4 (Ronquist et al. 2012). Two independent runs with four Markov Chain Monte Carlo simulations were performed for ten million iterations and sampled every 1000 iterations. The first $25 \%$ of samples were discarded as burn-in. Convergence of the Markov Chain Monte Carlo simulations was assessed by PSRF $\leq 0.01$ and ESS (effective sample size) value $>200$ using Tracer 1.4 (http://tree.bio.ed.ac.uk/software/tracer/). Genetic distances among all Leptobrachella samples were calculated in MEGA 6 using the uncorrected $p$-distance model, with pairwise deletion of gaps and missing data.

Table I. Collection localities, voucher data and GenBank numbers (16S rRNA) for all samples used in this study.

\begin{tabular}{|c|c|c|c|c|}
\hline ID & Ingroup & Collection Locality & Voucher No. & GenBank No. \\
\hline 1 & Leptobrachella aspera sp. nov. & Huanglianshan Nature Reserve, Lyuchun, Yunnan, China & SYS a007743 & MW046199 \\
\hline 2 & Leptobrachella aspera sp. nov. & Huanglianshan Nature Reserve, Lyuchun, Yunnan, China & SYS a007744 & MW046200 \\
\hline 3 & Leptobrachella aspera sp. nov. & Huanglianshan Nature Reserve, Lyuchun, Yunnan, China & SYS a007745 & MW046201 \\
\hline 4 & Leptobrachella aspera sp. nov. & Huanglianshan Nature Reserve, Lyuchun, Yunnan, China & SYS a007746 & MW046202 \\
\hline 5 & Leptobrachella dorsospina sp. nov. & Yushe Forest Park, Shuicheng, Guizhou, China & SYS a004961 & MW046194 \\
\hline 6 & Leptobrachella dorsospina sp. nov. & Yushe Forest Park, Shuicheng, Guizhou, China & SYS a004962 & MW046195 \\
\hline 7 & Leptobrachella dorsospina sp. nov. & Yushe Forest Park, Shuicheng, Guizhou, China & SYS a004973 & MW046196 \\
\hline 8 & Leptobrachella dorsospina sp. nov. & Yushe Forest Park, Shuicheng, Guizhou, China & SYS a004974 & MW046197 \\
\hline 9 & Leptobrachella dorsospina sp. nov. & Yushe Forest Park, Shuicheng, Guizhou, China & SYS a004975 & MW046198 \\
\hline 10 & Leptobrachella feii & Xiaoqiaogou Nature Reserve, Yunnan, China & KIZ032625 & MT302635 \\
\hline 11 & Leptobrachella feii & Xiaoqiaogou Nature Reserve, Yunnan, China & KIZ048894 & MT302634 \\
\hline 12 & Leptobrachella feii & Xiaoqiaogou Nature Reserve, Yunnan, China & KIZ048972 & MT302636 \\
\hline 13 & Leptobrachella feii & Xiaoqiaogou Nature Reserve, Yunnan, China & KIZ048973 & MT302637 \\
\hline 14 & Leptobrachella aerea & U Bo, Quang Binh, Vietnam & ZFMK 86362 & JN848409 \\
\hline 15 & Leptobrachella alpina & Huangcaoling, Jingdong, Yunnan, China & KIZ046816 & MH055866 \\
\hline 16 & Leptobrachella applebyi & Ngoc Linh, Kon Tum, Vietnam & AMS R 173778 & KR018108 \\
\hline 17 & Leptobrachella arayai & Mesilau, Sabah, Malaysia & BORNEENSIS 22931 & AB847558 \\
\hline 18 & Leptobrachella ardens & Kon Ka Kinh, Gia Lai, Vietnam & AMS R 176463 & KR018110 \\
\hline
\end{tabular}




\begin{tabular}{|c|c|c|c|c|}
\hline ID & Ingroup & Collection Locality & Voucher No. & GenBank No. \\
\hline 19 & Leptobrachella bidoupensis & Hon Giao, Lam Dong, Vietnam & NCSM 77321 & HQ902883 \\
\hline 20 & Leptobrachella bijie & Zhaozishan Nature Reserve, Guizhou, China & SYS a007320 & MK414539 \\
\hline 21 & Leptobrachella botsfordi & Fansipan, Lao Cai, Vietnam & AMS R 176540 & MH055953 \\
\hline 22 & Leptobrachella bourreti & Lao Cai, Vietnam & AMS R 177673 & KR018124 \\
\hline 23 & Leptobrachella chishuiensis & Chishui, Guizhou Province, China & CIBCS20190518047 & MT117053 \\
\hline 24 & Leptobrachella crocea & Kon Tum, Vietnam & AMS R 173740 & MH055954 \\
\hline 25 & Leptobrachella dringi & Gunung Mulu National Park, Sarawak, Malaysia & NMBE1056372 & KJ831298 \\
\hline 26 & Leptobrachella eos & Long Nai, Phongsaly, Laos & MNHN 2004.0274 & JN848452 \\
\hline 27 & Leptobrachella firthi & Ngoc Linh Nature Reserve, Kon Tum, Vietnam & AMS R 176524 & JQ739206 \\
\hline 28 & Leptobrachella flaviglandulosa & Xiaoqiaogou Nature Reserve, Yunnan, China & KIZ032626 & MT302633 \\
\hline 29 & Leptobrachella fritinniens & Base Camp of Mulu NP, Sarawak, Malaysia & KUHE 55371 & AB847557 \\
\hline 30 & Leptobrachella fuliginosa & Phetchaburi, Thailand & KUHE 20174 & LC201987 \\
\hline 31 & Leptobrachella gracilis & Camp 1 of Gunung Mulu NP, Sarawak, Malaysia & NMBE1056364 & KJ831300 \\
\hline 32 & Leptobrachella hamidi & Bukit Lanjak, Malaysia & KUHE 17545 & AB969286 \\
\hline 33 & Leptobrachella heteropus & Larut, Malaysia & KUHE 15486 & LC202005 \\
\hline 34 & Leptobrachella isos & Gia Lai, Vietnam & AMS R 176480 & KT824769 \\
\hline 35 & Leptobrachella kajangensis & Tioman, Malaysia & LSUHC 4431 & LC202001 \\
\hline 36 & Leptobrachella kalonensis & Song Luy, Binh Thuan, Vietnam & AMNH A191762 & KR018115 \\
\hline 37 & Leptobrachella kecil & Cameron, Malaysia & KUHE 52440 & LC202004 \\
\hline 38 & Leptobrachella khasiorum & Meghalaya, India & SDBDU 2009.329 & KY022303 \\
\hline 39 & Leptobrachella laui & Tai Mo Shan, Hongkong, China & SYS a002057 & KM014546 \\
\hline 40 & Leptobrachella liui & Guadun, Mt. Wuyi, Fujian, China & SYS a002479 & MH605574 \\
\hline 41 & Leptobrachella macrops & Phu Yen, Vietnam & PYU DTD-508 & MG787991 \\
\hline 42 & Leptobrachella maculosa & Phuoc Binh, Ninh Thuan, Vietnam & ZFMK 96600 & KR018120 \\
\hline 43 & Leptobrachella mangshanensis & Mangshan Nature Reserve, Hunan, China & MSZTC201701 & MG132196 \\
\hline 44 & Leptobrachella maoershanensis & Maoershan Nature Reserve, Guangxi, China & KIZ019385 & KY986930 \\
\hline 45 & Leptobrachella marmorata & Annah Rais, Padawan, Malaysia & KUHE 53192 & AB969287 \\
\hline 46 & Leptobrachella maura & Kinabalu, Malaysia & SP 21450 & AB847559 \\
\hline 47 & Leptobrachella melanoleuca & Srat Thani, Thailand & KUHE 19719 & LC201990 \\
\hline 48 & Leptobrachella melica & Virachey, Ratanakiri, Cambodia & MVZ 258197 & HM133599 \\
\hline 49 & Leptobrachella minima & Changdao, Thailand & KUHE 23733 & LC201980 \\
\hline 50 & Leptobrachella nahangensis & Na Hang Nature Reserve, Tuyen Quang, Vietnam & ROM 7035 & MH055853 \\
\hline 51 & Leptobrachella namdongensis & Thanh Hoa, Vietnam & VNUF A.2017.95 & MK965390 \\
\hline 52 & Leptobrachella niveimontis & Daxueshan Nature Reserve, Yunnan, China & KIZ015734 & MT302618 \\
\hline 53 & Leptobrachella nyx & Malipo, Yunnan, China & ROM 35606 & MH055814 \\
\hline 54 & Leptobrachella oshanensis & Mt. Emei, Sichuan, China & SYS a001830 & KM014810 \\
\hline 55 & Leptobrachella pallida & Gia Rich, Lam Dong, Vietnam & UNS00510 & KR018112 \\
\hline 56 & Leptobrachella pelodytoides & Tam Dao, Vinh Phu, Vietnam & MVZ 223642 & AY236798 \\
\hline 57 & Leptobrachella petrops & Tuyen Quang, Vietnam & VNMN:2016 A.06 & KY459998 \\
\hline 58 & Leptobrachella picta & Gunung Kinabalu National Park, Sabah, Malaysia & UNIMAS 8705 & KJ831295 \\
\hline 59 & Leptobrachella pluvialis & Sa Pa, Lao Cai, Vietnam & MNHN: 1999.5675 & JN848391 \\
\hline 60 & Leptobrachella puhoatensis & Nghe An, Vietnam & AMS R184852 & KY849588 \\
\hline 61 & Leptobrachella purpura & Yingjiang, Yunnan, China & SYS a006531 & MG520355 \\
\hline 62 & Leptobrachella purpuraventra & Wujing Nature Reserve, Guizhou, China & SYS a007277 & MK414518 \\
\hline 63 & Leptobrachella pyrrhops & Loc Bac, Lam Dong, Vietnam & ZMMU ABV-00176 & KP017576 \\
\hline 64 & Leptobrachella rowleyae & Son Tra, Da Nang, Vietnam & ITBCZ 4113 & MG682549 \\
\hline 65 & Leptobrachella sabahmontana & Mahua, Crocker, Malaysia & BORNEENSIS 12454 & AB847550 \\
\hline 66 & Leptobrachella shangsiensis & Shiwandashan, Guangxi, China & NHMG1401032 & MK095460 \\
\hline 67 & Leptobrachella sola & Terengganu, Malaysia & KUHE 52342 & LC202011 \\
\hline 68 & Leptobrachella suiyangensis & Suiyang, Guizhou, China & GZNU20180606002 & MK829648 \\
\hline 69 & Leptobrachella sungi & Bac Giang, Vietnam & ZMMU-NAP-02269 & MH055859 \\
\hline 70 & Leptobrachella tadungensis & Dak Nong, Vietnam & UNS00517 & KR018122 \\
\hline 71 & Leptobrachella tengchongensis & Tengchong, Yunnan, China & SYS a004598 & KU589209 \\
\hline 72 & Leptobrachella tuberosa & Kon Ka Kinh National Park, Gia Lai, Vietnam & ZMMU-NAP-02275 & MH055959 \\
\hline 73 & Leptobrachella ventripunctata & Xishuangbanna, Yunnan, China & SYS a001768 & KM014811 \\
\hline 74 & Leptobrachella wuhuangmontis & Mt. Wuhuang, Pubei, Guangxi, China & SYS a003485 & MH605577 \\
\hline 75 & Leptobrachella wulingensis & Tianzishan Nature Reserve, Hunan, China & CSUFT 200 & MT530317 \\
\hline 76 & Leptobrachella yingjiangensis & Yingjiang, Yunnan, China & SYS a006533 & MG520350 \\
\hline 77 & Leptobrachella yunkaiensis & Yunkaishan Nature Reserve, Guangdong, China & SYS a004663 & MH605584 \\
\hline 78 & Leptobrachella zhangyapingi & Chiang Mai, Thailand & KIZ07258 & MH055864 \\
\hline 79 & Leptobrachium tengchongense & Tengchong, Yunnan, China & SYS a004603 & KX066876 \\
\hline 80 & Oreolalax rhodostigmatus & Da Fang, Guizhou, China & CIB ZYCA746 & EF397248 \\
\hline
\end{tabular}




\section{Morphometrics}

Measurements followed Fei et al. (2009) and Rowley et al. (2013), and were taken with a digital caliper to the nearest $0.1 \mathrm{~mm}$. These measurements were as follows:

SVL snout-vent length (from tip of snout to vent);

HDL head length (from tip of snout to rear of jaws);

HDW head width (head width at commissure of jaws);

SNT snout length (from tip of snout to anterior corner of eye);

EYE eye diameter (diameter of exposed portion of eyeball);

IOD interorbital distance (minimum distance between upper eyelids);

IND internasal distance (distance between nares);

TMP tympanum diameter (horizontal diameter of tympanum);

TEY tympanum-eye distance (distance from anterior edge of tympanum to posterior corner of eye);

TIB tibia length (distance from knee to heel);

ML manus length (distance from tip of third digit to proximal edge of inner palmar tubercle);

PL pes length (distance from tip of fourth toe to proximal edge of the inner metatarsal tubercle);

LAHL length of lower arm and hand (distance from tip of the third finger to elbow); HLL hindlimb length (distance from tip of fourth toe to vent).

Sex was determined by the presence of internal vocal sac openings, and the presence of eggs in abdomen seen via external inspection.

All specimens were fixed in 10\% buffered formalin and later transferred to $70 \%$ ethanol for preservation, and deposited at the Museum of Biology, Sun Yat-sen University (SYS) and Chengdu Institute of Biology, the Chinese Academy of Sciences (CIB), China; tissue samples were preserved in 95\% ethanol for molecular studies.

Comparative morphological data of Leptobrachella species were obtained from examination of museum specimens (see Appendix 1) and from the references listed in Table 2. Due to the high likelihood of undiagnosed diversity within the genus (Rowley et al. 2016; Yang et al. 2016), where available, we rely on examination of topotypic material and/or original species descriptions.

\section{Results}

The BI analyses are shown in Fig. 2 with Bayesian posterior probabilities (BPP) for major nodes $>0.90$. Genetic distances among all Leptobrachella samples are given in the Suppl. material 1: Table S1. Comparative morphological data of all recognized Leptobrachella species occurring north of the Kra Isthmus are listed in Table 3. 
Table 2. Data sources of the 82 currently known species of the genus Leptobrachella.

\begin{tabular}{llc}
\hline ID & \multicolumn{1}{c}{ Leptobrachella species } & Literature \\
\hline $\mathbf{1}$ & L. aerea (Rowley, Stuart, Richards, Phimmachak \& Sivongxay, 2010) & Rowley et al. 2010c \\
$\mathbf{2}$ & L. alpina (Fei, Ye \& Li, 1990) & Fei et al. 2009, 2016 \\
$\mathbf{3}$ & L. applebyi (Rowley \& Cao, 2009) & Rowley and Cao 2009 \\
$\mathbf{4}$ & L. arayai (Matsui, 1997) & Matsui 1997 \\
$\mathbf{5}$ & L. ardens (Rowley, Tran, Le, Dau, Peloso, Nguyen, Hoang, Nguyen \& Ziegler, 2016) & Rowley et al. 2016 \\
$\mathbf{6}$ & L. baluensis Smith, 1931 & Dring 1983; Eto et al. 2016 \\
$\mathbf{7}$ & L. bijie Wang, Li, Li, Chen \& Wang, 2019 & Wang et al. 2019 \\
$\mathbf{8}$ & L. bidoupensis (Rowley, Le, Tran \& Hoang, 2011) & Rowley et al. 2011 \\
$\mathbf{9}$ & L. bondangensis Eto, Matsui, Hamidy, Munir \& Iskandar, 2018 & Eto et al. 2018 \\
$\mathbf{1 0}$ & L. botsfordi (Rowley, Dau \& Nguyen, 2013) & Rowley et al. 2013 \\
$\mathbf{1 1}$ & L. bourreti (Dubois, 1983) & Ohler et al. 2011 \\
$\mathbf{1 2}$ & L. brevicrus Dring, 1983 & Dring 1983; Eto et al. 2015 \\
$\mathbf{1 3}$ & L. crocea (Rowley, Hoang, Le, Dau \& Cao, 2010) & Rowley et al. 2010a \\
$\mathbf{1 4}$ & L. chishuiensis Li, Liu, Wei \& Wang, 2020 & Li et al. 2020a
\end{tabular}

15 L. dringi (Dubois, 1987)

16 L. eos (Ohler, Wollenberg, Grosjean, Hendrix, Vences, Ziegler \& Dubois, 2011)

17 L. feii Chen, Yuan \& Che, 2020

18 L. firthi (Rowley, Hoang, Dau, Le \& Cao, 2012)

19 L. fritinniens (Dehling \& Matsui, 2013)

20 L. fuliginosa (Matsui, 2006)

21 L. flaviglandulosa Chen, Wang \& Che, 2020

22 L. fusca Eto, Matsui, Hamidy, Munir \& Iskandar, 2018

23 L. gracilis (Günther, 1872)

24 L. hamidi (Matsui, 1997)

25 L. heteropus (Boulenger, 1900)

26 L. isos (Rowley, Stuart, Neang, Hoang, Dau, Nguyen \& Emmett, 2015)

27 L. itiokai Eto, Matsui \& Nishikawa, 2016

28 L. juliandringi Eto, Matsui \& Nishikawa, 2015

29 L. kajangensis (Grismer, Grismer \& Youmans, 2004)

30 L. kalonensis (Rowley, Tran, Le, Dau, Peloso, Nguyen, Hoang, Nguyen \& Ziegler, 2016)

31 L. kecil (Matsui, Belabut, Ahmad \& Yong, 2009)

32 L. khasiorum (Das, Tron, Rangad \& Hooroo, 2010)

33 L. lateralis (Anderson, 1871)

34 L. laui (Sung, Yang \& Wang, 2014)

35 L. liui (Fei \& Ye, 1990)

36 L. macrops (Duong, Do, Ngo, Nguyen \& Poyarkov, 2018)

37 L. maculosa (Rowley, Tran, Le, Dau, Peloso, Nguyen, Hoang, Nguyen \& Ziegler, 2016)

38 L. mangshanensis (Hou, Zhang, Hu, Li, Shi, Chen, Mo \& Wang, 2018)

39 L. maoershanensis (Yuan, Sun, Chen, Rowley \& Che, 2017)

40 L. marmorata (Matsui, Zainudin \& Nishikawa, 2014)

41 L. maura (Inger, Lakim, Biun \& Yambun, 1997)

42 L. melanoleuca (Matsui, 2006)

43 L. melica (Rowley, Stuart, Neang \& Emmett, 2010)

44 L. minima (Taylor, 1962)

45 L. mjobergi Smith, 1925

46 L. nahangensis (Lathrop, Murphy, Orlov \& Ho, 1998)

47 L. natunae (Günther, 1895)

48 L. namdongensis Hoang, Nguyen, Luu, Nguyen \& Jiang, 2019

49 L. neangi Stuart \& Rowley, 2020

50 L. niveimontis Chen, Poyarkov, Yuan \& Che, 2020

51 L. nokrekensis (Mathew \& Sen, 2010)

52 L. nyx (Ohler, Wollenberg, Grosjean, Hendrix, Vences, Ziegler \& Dubois, 2011)

53 L. oshanensis (Liu, 1950)

54 L. pallida (Rowley, Tran, Le, Dau, Peloso, Nguyen, Hoang, Nguyen \& Ziegler, 2016)

55 L. palmata Inger \& Stuebing, 1992

56 L. parva Dring, 1983

57 L. pelodytoides (Boulenger, 1893)

Inger et al. 1995; Matsui and Dehling 2012

Ohler et al. 2011

Chen et al. 2020

Rowley et al. 2012

Dehling and Matsui 2013

Matsui 2006

Chen et al. 2020

Eto et al. 2018

Günther 1872; Dehling 2012b

Matsui 1997

Boulenger 1900

Rowley et al. 2015a

Eto et al. 2016

Eto et al. 2015

Grismer et al. 2004

Rowley et al. 2016

Matsui et al. 2009

Das et al. 2010

Anderson 1871; Humtsoe et al. 2008

Sung et al. 2014

Fei et al. 2009; Sung et al. 2014

Duong et al. 2018

Rowley et al. 2016

Hou et al. 2018

Yuan et al. 2017

Matsui et al. 2014b

Inger et al. 1997

Matsui 2006

Rowley et al. 2010b

Taylor 1962; Ohler et al. 2011 Eto et al. 2015

Lathrop et al. 1998 Günther 1895

Hoang et al. 2019

Stuart and Rowley 2020

Chen et al. 2020

Mathew and Sen 2010

Ohler et al. 2011

Fei et al. 2009, 2016

Rowley et al. 2016

Inger and Stuebing 1992

Dring 1983

Boulenger 1893; Ohler et al. 2011 


\begin{tabular}{llc}
\hline ID & \multicolumn{1}{c}{ Leptobrachella species } & Literature \\
\hline $\mathbf{5 8}$ & L. petrops (Rowley, Dau, Hoang, Le, Cutajar \& Nguyen, 2017) & Rowley et al. 2017a \\
$\mathbf{5 9}$ & L. picta (Malkmus, 1992) & Malkmus 1992 \\
$\mathbf{6 0}$ & L. platycephala (Dehling, 2012) & Dehling 2012a \\
$\mathbf{6 1}$ & L. pluvialis (Ohler, Marquis, Swan \& Grosjean, 2000) & Ohler et al. 2000, 2011 \\
$\mathbf{6 2}$ & L. puhoatensis (Rowley, Dau \& Cao, 2017) & Rowley et al. 2017b \\
$\mathbf{6 3}$ & L. purpura (Yang, Zeng \& Wang, 2018) & Yang et al. 2018 \\
$\mathbf{6 4}$ & L. purpuraventra Wang, Li, Li, Chen \& Wang, 2019 & Wang et al. 2019 \\
$\mathbf{6 5}$ & L. pyrrhops (Poyarkov, Rowley, Gogoleva, Vassilieva, Galoyan \& Orlov, 2015) & Poyarkov et al. 2015 \\
$\mathbf{6 6}$ & L. rowleyae (Nguyen, Poyarkov, Le, Vo, Ninh, Duong, Murphy \& Sang, 2018) & Nguyen et al. 2018 \\
$\mathbf{6 7}$ & L. sabahmontana (Matsui, Nishikawa \& Yambun, 2014) & Matsui et al. 2014a \\
$\mathbf{6 8}$ & L. serasanae Dring, 1983 & Dring 1983 \\
$\mathbf{6 9}$ & L. shangsiensis Chen, Liao, Zhou \& Mo, 2019 & Chen et al. 2019 \\
$\mathbf{7 0}$ & L. sola (Matsui, 2006) & Matsui 2006 \\
$\mathbf{7 1}$ & L. suiyangensis Luo, Xiao, Gao \& Zhou, 2020 & Luo et al. 2020 \\
$\mathbf{7 2}$ & L. sungi (Lathrop, Murphy, Orlov \& Ho, 1998) & Lathrop et al. 1998 \\
$\mathbf{7 3}$ & L. tadungensis (Rowley, Tran, Le, Dau, Peloso, Nguyen, Hoang, Nguyen \& Ziegler, 2016) & Rowley et al. 2016 \\
$\mathbf{7 4}$ & L. tamdil (Sengupta, Sailo, Lalremsanga, Das \& Das, 2010) & Sengupta et al. 2010 \\
$\mathbf{7 5}$ & L. tengchongensis (Yang, Wang, Chen \& Rao, 2016) & Yang et al. 2016 \\
$\mathbf{7 6}$ & L. tuberosa (Inger, Orlov \& Darevsky, 1999) & Inger et al. 1999 \\
77 & L. ventripunctata (Fei, Ye \& Li, 1990) & Fei et al. 2009, 2016 \\
$\mathbf{7 8}$ & L. wuhuangmontis Wang, Yang \& Wang, 2018 & Wang et al. 2018 \\
$\mathbf{7 9}$ & L. wulingensis Qian, Xia, Cao, Xiao \& Yang, 2020 & Qian et al. in publication \\
$\mathbf{8 0}$ & L. yingjiangensis (Yang, Zeng \& Wang, 2018) & Yang et al. 2018 \\
$\mathbf{8 1}$ & L. yunkaiensis Wang, Li, Lyu \& Wang, 2018 & Wang et al. 2018 \\
$\mathbf{8 2}$ & L. zhangyapingi (Jiang, Yan, Suwannapoom, Chomdej \& Che, 2013) & Jiang et al. 2013 \\
\hline
\end{tabular}

As shown by the phylogenetic result, Leptobrachella samples from Huanglianshan Nature Reserve are clustered in a distinct and robust monophyletic lineage with strong support (BPP 1.00). This lineage forms the sister taxon to L. feii occurring in Xiaoqiaogou Nature Reserve (BPP 1.00). The genetic distances between these two lineages are 3.0-3.4\%, which is significantly larger than that among other recognized species (e.g., $p$-distance $2.6 \%$ between $L$. liui and L. mangshanensis). Detailed morphological examination also reveals a combination of characters that distinguish the specimens of the unnamed lineage from L. feii and other known congeners (see taxonomic comparison below). Therefore, based on the molecular and morphological differences, the population from Huanglianshan Nature Reserve is proposed as a new species, Leptobrachella aspera sp. nov.

Samples of the other unnamed lineage from Yushe Forest Park, cluster in another distinct and robust monophyletic lineage with strong support (BPP 1.00). This lineage is close to several species occurring in southwestern China, but its specific placement remains unresolved due to the insufficient support values. The smallest genetic distance between this lineage and another congener is $3.5 \%$ (vs. L. purpuraventra), which is significantly larger than that between other recognized species (e.g., $p$-distance $2.6 \%$ between $L$. liui and L. mangshanensis). Detailed morphological examination also reveals a combination of characteristics distinguishing the specimens of this lineage from all known congeners (see taxonomic comparison below). Therefore, based on the molecular and morphological differences, the population from Yushe Forest Park is proposed as a new species, Leptobrachella dorsospina sp. nov. 


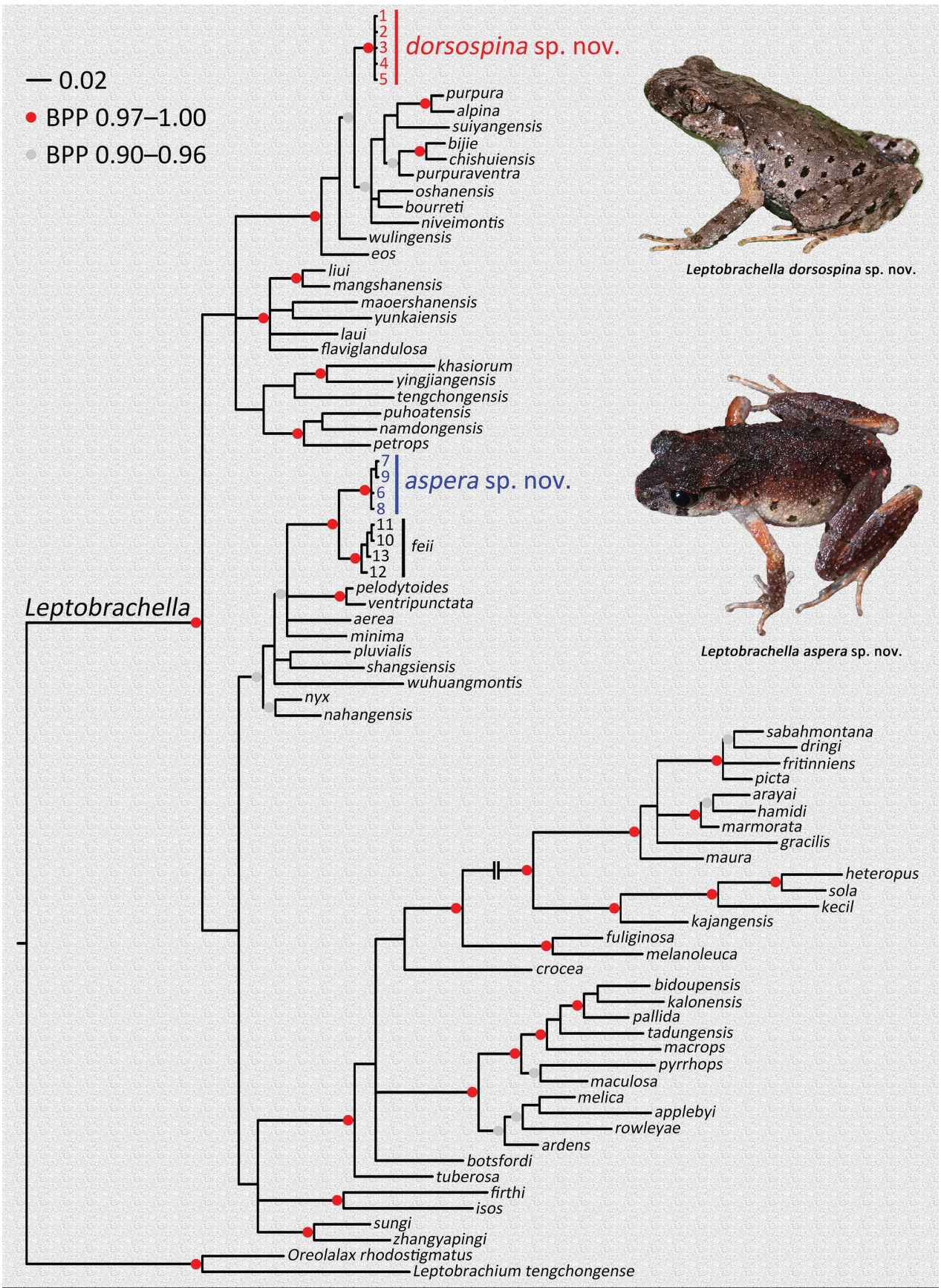

Figure 2. Bayesian Inference tree. The Bayesian posterior probabilities (BPP) $>0.90$ were retained. 
Table 3. Comparisons of selected diagnostic characters for the new species described herein and congeners occurring north of the Kra Isthmus (modified from Rowley et al. 2017; Wang et al. 2019; Chen et al. 2020).

\begin{tabular}{|c|c|c|c|c|c|c|}
\hline $\begin{array}{c}\text { Leptobrachella } \\
\text { species }\end{array}$ & $\begin{array}{l}\text { Male SVL } \\
(\mathrm{mm})\end{array}$ & $\begin{array}{c}\text { Black spots } \\
\text { on flanks }\end{array}$ & Toe webbing & $\begin{array}{c}\text { Toe } \\
\text { fringes }\end{array}$ & Ventral coloration & Dorsal skin texture \\
\hline L. aspera sp. nov. & 22.4 & Present & Rudimentary & Narrow & $\begin{array}{l}\text { Creamy white with distinct dark } \\
\text { patches on chest and abdomen }\end{array}$ & $\begin{array}{l}\text { Rough with dense conical } \\
\text { granules, tubercles, and } \\
\text { glandular folds }\end{array}$ \\
\hline $\begin{array}{l}\text { L. dorsospina } \\
\text { sp. nov. }\end{array}$ & $28.7-30.5$ & Present & Rudimentary & Narrow & $\begin{array}{l}\text { Greyish white with black spots and } \\
\text { orange pigmentations }\end{array}$ & $\begin{array}{l}\text { Rough with dense conical } \\
\text { granules, tubercles, glandular } \\
\text { folds, and conical spines }\end{array}$ \\
\hline L. aerea & $25.1-28.9$ & Absent & Rudimentary & Wide & $\begin{array}{l}\text { Near immaculate creamy white, } \\
\text { brown speckles on margins }\end{array}$ & Finely tuberculate \\
\hline L. alpina & $24.0-26.4$ & Present & Rudimentary & $\begin{array}{l}\text { Wide in } \\
\text { males }\end{array}$ & Creamy-white with dark spots & $\begin{array}{l}\text { Relatively smooth, some with } \\
\text { small warts }\end{array}$ \\
\hline L. applebyi & $19.6-22.3$ & Present & Rudimentary & Absent & Reddish brown with white speckles & Smooth \\
\hline L. ardens & $21.3-24.7$ & Present & Absent & Absent & Reddish brown with white speckles & Smooth, finely shagreened \\
\hline L. bidoupensis & $18.5-25.4$ & Present & Rudimentary & Weak & Reddish brown with white speckles & Smooth \\
\hline L. bijie & $29.0-30.4$ & Present & Rudimentary & Narrow & $\begin{array}{c}\text { White with distinct nebulous } \\
\text { greyish speckles on chest and } \\
\text { ventrolateral flanks }\end{array}$ & Shagreened and granular \\
\hline L. botsfordi & $29.1-32.6$ & Absent & Rudimentary & Narrow & Reddish brown with white speckles & Shagreened \\
\hline L. bourreti & $28.0-36.2$ & Present & Rudimentary & Weak & Creamy white & $\begin{array}{l}\text { Relatively smooth, some with } \\
\text { small warts }\end{array}$ \\
\hline L. crocea & $22.2-27.3$ & Absent & Rudimentary & Absent & Bright orange & Highly tuberculate \\
\hline L. chishuiensis & $30.8-33.4$ & Present & Rudimentary & Narrow & $\begin{array}{l}\text { White with distinct nebulous } \\
\text { greyish speckles on chest and } \\
\text { ventrolateral flanks }\end{array}$ & Shagreened and granular \\
\hline L. eos & $33.1-34.7$ & Absent & Rudimentary & Wide & Creamy white & Shagreened \\
\hline L. feii & $21.5-22.8$ & Present & Rudimentary & Narrow & Creamy white with black blotches & $\begin{array}{l}\text { Shagreened with small } \\
\text { tubercles } \\
\text { and ridge }\end{array}$ \\
\hline L. firthi & $26.4-29.2$ & Absent & Rudimentary & $\begin{array}{l}\text { Wide in } \\
\text { males }\end{array}$ & Creamy white & Shagreened with fine tubercles \\
\hline L. flaviglandulosa & $23.0-27.0$ & Present & $\begin{array}{c}\text { Poorly } \\
\text { developed }\end{array}$ & Narrow & $\begin{array}{l}\text { Whitish with black speckles on } \\
\text { margins }\end{array}$ & $\begin{array}{l}\text { Shagreened with yellowish- } \\
\text { brown tubercles }\end{array}$ \\
\hline L. fuliginosa & $28.2-30.0$ & Present & Rudimentary & Weak & White with brown dusting & $\begin{array}{l}\text { Nearly smooth with few } \\
\text { tubercles }\end{array}$ \\
\hline L. isos & $23.7-27.9$ & Absent & Rudimentary & $\begin{array}{l}\text { Wide in } \\
\text { males }\end{array}$ & $\begin{array}{l}\text { Creamy white with white dusting } \\
\text { on margins }\end{array}$ & $\begin{array}{c}\text { Mostly smooth, females more } \\
\text { tuberculate }\end{array}$ \\
\hline L. kalonensis & $25.8-30.6$ & Present & Absent & Absent & Pale, speckled brown & Smooth \\
\hline L. khasiorum & $24.5-27.3$ & Present & Rudimentary & Wide & Creamy white & Isolated, scattered tubercles \\
\hline L. laui & $24.8-26.7$ & Present & Rudimentary & Wide & $\begin{array}{l}\text { Creamy white with dark brown } \\
\text { dusting on margins }\end{array}$ & Round granular tubercles \\
\hline L. liui & $23.0-28.7$ & Present & Rudimentary & Wide & $\begin{array}{l}\text { Creamy white with dark brown } \\
\text { spots on chest and margins }\end{array}$ & $\begin{array}{l}\text { Round granular tubercles with } \\
\text { glandular folds }\end{array}$ \\
\hline L. lateralis & $26.9-28.3$ & Present & Rudimentary & Absent & Creamy white & Roughly granular \\
\hline L. macrops & $28.0-29.3$ & Present & Rudimentary & Absent & Greyish violet with white speckles & $\begin{array}{l}\text { Roughly granular with larger } \\
\text { tubercles }\end{array}$ \\
\hline L. maculosa & $24.2-26.6$ & Present & Absent & Absent & Brown with few white speckles & Mostly smooth \\
\hline L. mangshanensis & $22.2-27.8$ & Present & Rudimentary & Weak & White speckles on throat and belly & Nearly smooth \\
\hline L. maoershanensis & $25.2-30.4$ & Present & Rudimentary & Narrow & $\begin{array}{c}\text { Creamy white chest and belly with } \\
\text { irregular black spots }\end{array}$ & With longitudinal folds \\
\hline L. melica & $19.5-22.7$ & Present & Rudimentary & Absent & Reddish brown with white speckles & Smooth \\
\hline L. minima & $25.7-31.4$ & Present & Rudimentary & Absent & Creamy white & Smooth \\
\hline L. nahangensis & 40.8 & Present & Rudimentary & Absent & $\begin{array}{c}\text { Creamy white with light speckles } \\
\text { on throat and chest }\end{array}$ & Smooth \\
\hline L. niveimontis & $22.5-23.6$ & Present & Rudimentary & Narrow & Marbling with black speckles & $\begin{array}{l}\text { Relatively smooth with small } \\
\text { tubercles }\end{array}$ \\
\hline L. nokrekensis & $26.0-33.0$ & Present & Rudimentary & Unknown & Creamy white & $\begin{array}{l}\text { Tubercles and longitudinal } \\
\text { folds }\end{array}$ \\
\hline L. $n y x$ & $26.7-32.6$ & Present & Rudimentary & Absent & Creamy white with brown margins & Rounded tubercles \\
\hline L. namdongensis & 30.9 & Present & Rudimentary & Absent & $\begin{array}{c}\text { Immaculate white, chest and } \\
\text { belly with dark specking on outer } \\
\text { margins }\end{array}$ & $\begin{array}{l}\text { Low, round tubercles, more } \\
\text { dense } \\
\text { in posterior part of the back }\end{array}$ \\
\hline
\end{tabular}




\begin{tabular}{|c|c|c|c|c|c|c|}
\hline $\begin{array}{c}\begin{array}{c}\text { Leptobrachella } \\
\text { species }\end{array} \\
\end{array}$ & \begin{tabular}{|c|}
$\begin{array}{c}\text { Male SVL } \\
(\mathrm{mm})\end{array}$ \\
\end{tabular} & $\begin{array}{c}\text { Black spots } \\
\text { on flanks }\end{array}$ & Toe webbing & $\begin{array}{c}\text { Toe } \\
\text { fringes }\end{array}$ & Ventral coloration & Dorsal skin texture \\
\hline L. neangi & - & Present & $\begin{array}{l}\text { Weak (in } \\
\text { females) }\end{array}$ & $\begin{array}{l}\text { Absent (in } \\
\text { females) }\end{array}$ & $\begin{array}{l}\text { Light purplish gray with dark } \\
\text { brown mottling on throat }\end{array}$ & $\begin{array}{l}\text { Small, irregular bumps and } \\
\text { ridges }\end{array}$ \\
\hline L. oshanensis & $26.6-30.7$ & Present & Absent & Absent & $\begin{array}{c}\text { Whitish with no markings or only } \\
\text { small, light grey spots }\end{array}$ & $\begin{array}{c}\text { Smooth with few glandular } \\
\text { ridges }\end{array}$ \\
\hline L. pallida & $24.5-27.7$ & Absent & Absent & Absent & Reddish brown with white speckles & Tuberculate \\
\hline L. pelodytoides & $27.5-32.3$ & Present & Wide & Narrow & Whitish & Small, smooth warts \\
\hline L. petrops & $23.6-27.6$ & Absent & Absent & Narrow & Immaculate creamy white & Highly tuberculate \\
\hline L. pluvialis & $21.3-22.3$ & Present & Rudimentary & Absent & $\begin{array}{l}\text { Dirty white with dark brown } \\
\text { marbling }\end{array}$ & $\begin{array}{l}\text { Smooth, flattened tubercles } \\
\text { on flanks }\end{array}$ \\
\hline L. puhoatensis & $24.2-28.1$ & Present & Rudimentary & Narrow & Reddish brown with white dusting & With longitudinal skin ridges \\
\hline L. purpura & $25.0-27.5$ & Present & Rudimentary & Wide & $\begin{array}{c}\text { Dull white with indistinct grey } \\
\text { dusting }\end{array}$ & Shagreen with small tubercles \\
\hline L. purpuraventra & $27.3-29.8$ & Present & Rudimentary & Narrow & $\begin{array}{l}\text { Grey purple with distinct nebulous } \\
\text { greyish speckles on chest and } \\
\text { ventrolateral flanks }\end{array}$ & Shagreened with granules \\
\hline L. pyrrhops & $30.8-34.3$ & Present & Rudimentary & Absent & Reddish brown with white speckles & Slightly shagreened \\
\hline L. rowleyae & $23.4-25.4$ & Present & Absent & Absent & $\begin{array}{c}\text { Pinkish milk-white to light brown } \\
\text { chest and belly with numerous } \\
\text { white speckles }\end{array}$ & $\begin{array}{c}\text { Smooth with numerous tiny } \\
\text { tubercles }\end{array}$ \\
\hline L. suiyangensis & $28.7-29.7$ & Present & Rudimentary & Narrow & $\begin{array}{c}\text { Yellowish creamy-white with } \\
\text { marble texture chest and belly or } \\
\text { with irregular light brown speckles }\end{array}$ & Shagreen with small granules \\
\hline L. sungi & $48.3-52.7$ & $\begin{array}{c}\text { Absent or } \\
\text { small }\end{array}$ & Wide & Weak & White & Granular \\
\hline L. tadungensis & $23.3-28.2$ & Present & Absent & Absent & Reddish brown with white speckles & Smooth \\
\hline L. tamdil & 32.3 & Present & Wide & Wide & White & Weakly tuberculate \\
\hline L. tengchongensis & $23.9-26.0$ & Present & Rudimentary & Narrow & White with dark brown blotches & $\begin{array}{l}\text { Shagreened with small } \\
\text { tubercles }\end{array}$ \\
\hline L. tuberosa & $24.4-29.5$ & Absent & Rudimentary & Absent & White with small grey spots/streaks & Highly tuberculate \\
\hline L. ventripunctata & $25.5-28.0$ & Present & Rudimentary & Absent & $\begin{array}{l}\text { Chest and belly with dark brown } \\
\text { spots }\end{array}$ & Longitudinal skin ridges \\
\hline $\begin{array}{l}\text { L. } \\
\text { wuhuangmontis }\end{array}$ & $25.6-30.0$ & Present & Rudimentary & Narrow & $\begin{array}{c}\text { Greyish white mixed by tiny white } \\
\text { and black dots }\end{array}$ & $\begin{array}{l}\text { Rough, scattered with dense } \\
\text { conical tubercles }\end{array}$ \\
\hline L. wulingensis & $22.7-30.5$ & Present & Rudimentary & Narrow & $\begin{array}{l}\text { Translucent creamy white, with } \\
\text { distinct or indistinct brown } \\
\text { speckles at margins }\end{array}$ & $\begin{array}{c}\text { Shagreened with sparse large } \\
\text { warts, some with longitudinal } \\
\text { ridges }\end{array}$ \\
\hline L. yingjiangensis & $25.7-27.6$ & Present & Rudimentary & Wide & $\begin{array}{l}\text { Creamy white with dark brown } \\
\text { flecks on chest and margins }\end{array}$ & $\begin{array}{l}\text { Shagreened with small } \\
\text { tubercles }\end{array}$ \\
\hline L. yunkaiensis & $25.9-29.3$ & Present & Rudimentary & Wide & $\begin{array}{l}\text { Belly pink with distinct or } \\
\text { indistinct speckles }\end{array}$ & $\begin{array}{l}\text { Shagreened with short skin } \\
\text { ridges and raised warts }\end{array}$ \\
\hline L. zhangyapingi & $45.8-52.5$ & Absent & Rudimentary & Wide & Creamy-white with brown margins & $\begin{array}{c}\text { Mostly smooth with distinct } \\
\text { tubercles }\end{array}$ \\
\hline
\end{tabular}

\section{Taxonomic accounts}

\section{Leptobrachella aspera Wang, Lyu, Qi \& Wang, sp. nov.}

http://zoobank.org/4919B18E-B0D0-4329-90BF-8AC77280D263

Fig. 3

Type material. Holotype. SYS a007743, adult male, collected by Jian Wang, Yao Li and Yu-Long Li on 31 May 2019 from Huanglianshan Nature Reserve $\left(22.89^{\circ} \mathrm{N}\right.$, 102.29E; ca. 1930 m a.s.l.), Lyuchun County, Yunnan Province, China.

Paratypes $(\mathrm{N}=3)$. Three adult females, SYS a007744-7745, SYS a007746/ CIB116080, the same collection data as the holotype.

Diagnosis. (1) Small size (SVL $22.4 \mathrm{~mm}$ in a single adult male, 25.0-26.4 in three adult females), (2) dorsal skin rough, with dense conical granules, tubercles 


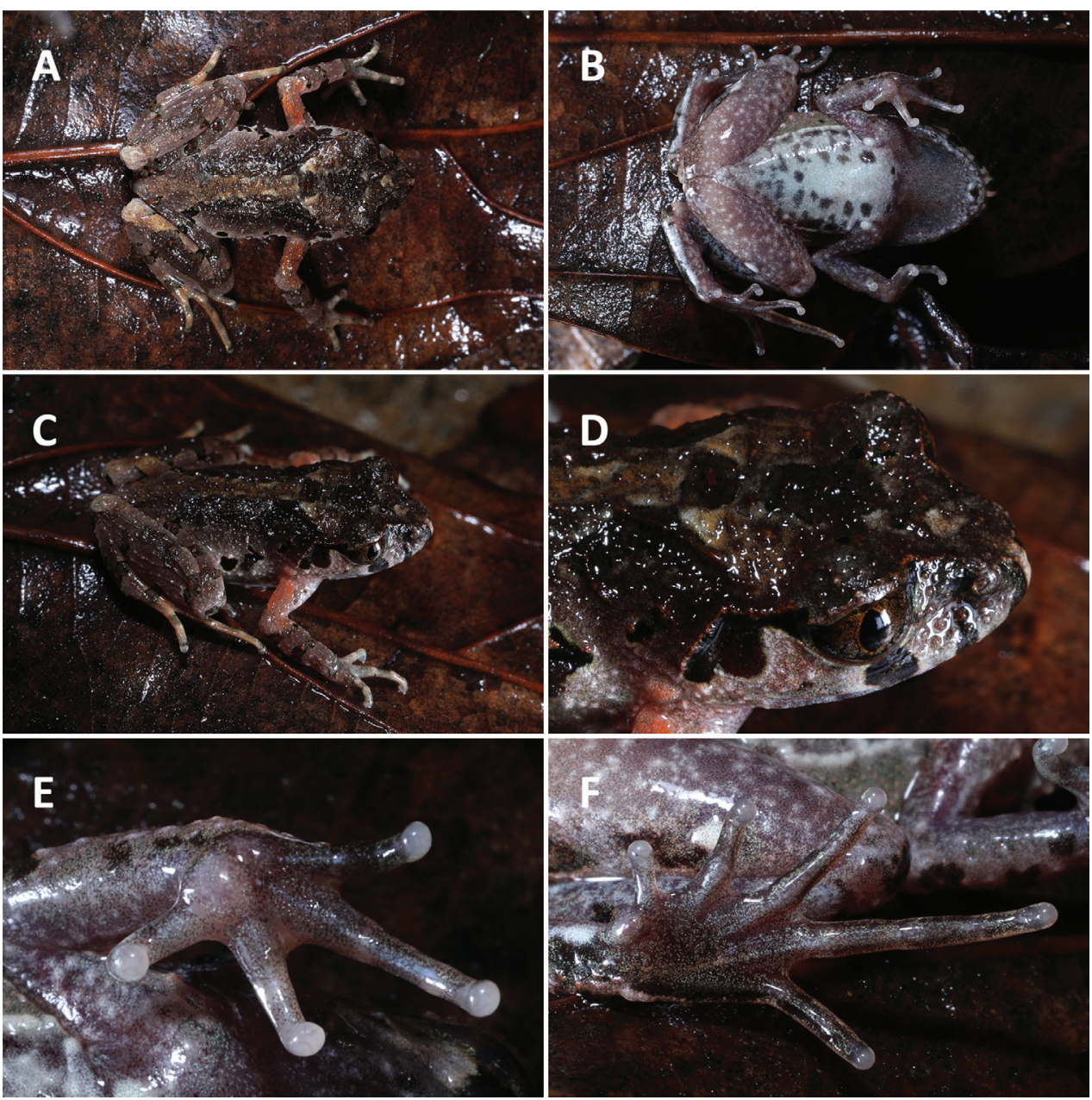

Figure 3. Morphological features in life. Leptobrachella aspera sp. nov., holotype SYS a007743.

and glandular folds, (3) iris bicolored, amber on upper half and silver on lower half, (4) tympanum distinctly discernible, distinct black supratympanic line present, (5) absence of webbing and lateral fringes on fingers, toes with rudimentary webbing and narrow lateral fringes both in males and females, (6) longitudinal ridges under toes not interrupted at the articulations, (7) relative finger lengths $\mathrm{I}<\mathrm{IV}<\mathrm{II}<\mathrm{III}$, relative toe length $\mathrm{I}<\mathrm{II}<\mathrm{V}<\mathrm{III}<\mathrm{IV}$, (8) heels just meeting, tibia-tarsal articulation reaches the region between middle of eye to anterior corner of eye, (9) dorsum greyish brown to yellowish brown grounding, with small light orange granules and distinct darker brown markings scattered with irregular light orange or greyish white pigmentations, (10) flanks with several enlarged dark patches with light yellowish green margin, (11) ventral surface creamy white, with distinct regular dark patches on chest and abdomen. 
Comparison. From the 26 known congeners of the genus Leptobrachella occurring south of the Kra Isthmus, the presence of supra-axillary and ventrolateral glands, can easily distinguish $L$. aspera sp. nov. from $L$. arayai, $L$. dringi, $L$. fritinniens, $L$. gracilis, L. hamidi, L. heteropus, L. kajangensis, L. kecil, L. marmorata, L. melanoleuca, L. maura, L. picta, L. platycephala, L. sabahmontana and L. sola, all of which lack the supra-axillary and ventrolateral glands; and by the significantly larger body size, SVL $22.4 \mathrm{~mm}$ in a single male, $L$. aspera sp. nov. differs from the smaller L. baluensis $(14.9-15.9 \mathrm{~mm}$ in males), L. brevicrus (17.1-17.8 $\mathrm{mm}$ in males), L. bondangensis (17.8 $\mathrm{mm}$ in male), L. fusca (16.3 $\mathrm{mm}$ in male), L. itiokai (15.2-16.7 $\mathrm{mm}$ in males), L. juliandringi (17.0$17.2 \mathrm{~mm}$ in males), L. mjobergi (15.7-19.0 $\mathrm{mm}$ in males), L. natunae (17.6 $\mathrm{mm}$ in one adult male), $L$. parva (15.0-16.9 mm in males), L. palmata (14.4-16.8 mm in males), and $L$. serasanae ( $16.9 \mathrm{~mm}$ in female).

Leptobrachella aspera sp. nov. is recovered as a sister taxon to L. feii in the phylogenetic tree (Fig. 2). However, the new species can be distinguished from L. feii by the following morphological characters: head relatively short, HDL/SVL $0.33-0.35$ (vs. head relatively long, HDL/SVL 0.38-0.43); distinct regular dark patches on skin of chest and abdomen (vs. irregular black blotches scattered on skin of chest and belly); color of upper half of iris amber (vs. color of upper half of iris lighter, golden orange); ventrolateral glands forming an non-continuous line (vs. ventrolateral glands forming a continuous line); relative finger lengths I $<$ IV $<$ II $<$ III (vs. relative finger lengths II $<$ I $<$ IV $<$ III); tibio-tarsal articulation of adpressed limb reaching the region between middle of eye to anterior corner of eye (vs. tibio-tarsal articulation of adpressed limb reaching beyond eye).

While Leptobrachella pluvialis is distributed in the same mountain range on the Vietnamese side and possesses similar body size (Ohler et al. 2000), it can be separated from L. aspera sp. nov. by the following characters: (1) smooth dorsal skin with flattened tubercles on flanks (vs. rough dorsal skin with dense conical granules in L. aspera sp. nov.), (2) absence of webbing on toes (vs. rudimentary webbing on toes in L. aspera sp. nov.), and (3) relatively longer hindlimbs, the tibia-tarsal articulation reaching to the nostril (vs. relatively shorter hindlimbs, the tibia-tarsal articulation reaching the region between middle of eye to anterior corner of eye in L. aspera sp. nov.).

For the remaining 54 members of the genus Leptobrachella, in having SVL $22.4 \mathrm{~mm}$ in a single male, $L$. aspera sp. nov. differs from the larger $L$. aerea (25.1-28.9 in males), L. alpina (24.0-28.9 mm in males), L. bijie (29.0-30.4 mm in males), L. botsfordi (29.1-32.6 $\mathrm{mm}$ in males), L. bourreti (28.0-36.2 $\mathrm{mm}$ in males), L. chishuiensis (30.833.4 in males), L. eos (33.1-34.7 mm in males), L. firthi (26.4-29.2 $\mathrm{mm}$ in males), L. flaviglandulosa (23.0-27.0 $\mathrm{mm}$ in males), L. fuliginosa (28.2-30.0 $\mathrm{mm}$ in males), L. isos (23.7-27.9 $\mathrm{mm}$ in males), L. kalonensis (25.8-30.6 $\mathrm{mm}$ in males), L. khasiorum (24.5-27.3 mm in males), L. laui (24.8-26.7 $\mathrm{mm}$ in males), L. lateralis (26.9-28.3 mm in males), L. macrops (28.0-29.3 mm in males), L. maculosa (24.2-26.6 $\mathrm{mm}$ in males), L. minima (25.7-31.4 $\mathrm{mm}$ in males), L. nahangensis ( $40.8 \mathrm{~mm}$ in male), L. nokrekensis (26.0-33.0 mm in males), L. nyx (26.7-32.6 mm in males), L. neangi (30.9 $\mathrm{mm}$ in male), L. namdongensis (30.9 $\mathrm{mm}$ in male), L. oshanensis (26.6-30.7 $\mathrm{mm}$ in males), L. pallida 
(24.5-27.7 $\mathrm{mm}$ in males), L. pelodytoides (27.5-32.3 $\mathrm{mm}$ in males), L. petrops (23.6$27.6 \mathrm{~mm}$ in males), $L$. puhoatensis (24.2-28.1 mm in males), L. purpura (25.0-27.5 mm in males), L. purpuraventra (27.3-29.8 $\mathrm{mm}$ in males), L. pyrrhops $(30.8-34.3 \mathrm{~mm}$ in males), L. rowleyae (23.4-25.4 mm in males), L. suiyangensis (28.7-29.7 $\mathrm{mm}$ in males), L. sungi (48.3-52.7 $\mathrm{mm}$ in males), L. tadungensis (23.3-28.2 $\mathrm{mm}$ in males), L. tamdil (32.3 $\mathrm{mm}$ in male), L. tengchongensis (23.9-26.0 $\mathrm{mm}$ in males), L. tuberosa (24.4$29.5 \mathrm{~mm}$ in males), L. ventripunctata (25.5-28.0 $\mathrm{mm}$ in males), L. wuhuangmontis (25.6-30.0 $\mathrm{mm}$ in males), L. yingjiangensis (25.7-27.6 mm in males), L. yunkaiensis (25.9-29.3 $\mathrm{mm}$ in males), and L. zhangyapingi (45.8-52.5 $\mathrm{mm}$ in males). By presence of black spots on flanks, the new species can be distinguished from $L$. crocea, versus absence of black spots on flanks; by rudimentary webbing on toes, the new species can be distinguished from $L$. ardens, versus absence of webbing on toes; by narrow lateral fringes on toes, the new species can be distinguished from $L$. applebyi, $L$. ardens, $L$. crocea, and L. melica, all having no lateral fringes on toes, and from L. liui, having wide lateral fringes on toes; by the creamy white ventral coloration and distinct regular dark patches on the chest and abdomen, the new species can be distinguished from L. applebyi, L. ardens, L. bidoupensis, and L. melica, all having reddish brown ventral coloration with white specks, from $L$. crocea, having bright orange ventral coloration, from $L$. mangshanensis, lacking dark skin patches on the chest and abdomen, from L. liui, having creamy white ventral coloration with dark brown spots on the chest and margins, and from $L$. niveimontis, having marbling ventral coloration with black speckling; by rough dorsal skin with dense conical granules, tubercles and glandular folds, the new species can be distinguished from L. applebyi, L. ardens, L. bidoupensis, L. mangshanensis, L. melica, and L. niveimontis, all having smooth dorsal skin.

Description of holotype. Adult male. Body size small, SVL $22.4 \mathrm{~mm}$. Head length slightly larger than head width, HDW/HDL 0.99 ; snout slightly protruding, projecting slightly beyond margin of the lower jaw; nostril closer to snout than eye; canthus rostralis gently rounded; loreal region slightly concave; interorbital space flat, internarial distance greater than interorbital distance, IND/IOD 1.07; pineal ocellus absent; pupil vertical; snout length longer than eye diameter, SNT/EYE 1.26; tympanum distinct, rounded, and slightly concave, diameter smaller than that of the eye and larger than tympanum-eye distance, TMP/EYE 0.52 and TEY/TMP 0.44; upper margin of tympanum in contact with supratympanic ridge; distinct black supratympanic line present; vomerine teeth absent; vocal sac openings slit-like, paired, located posterolaterally on floor of mouth in close proximity to the margins of the mandible; tongue deeply notched posteriorly; supratympanic ridge distinct, extending from posterior corner of eye to supra-axillary gland.

Tips of fingers rounded, slightly swollen; relative finger lengths I $<$ IV $<$ II $<$ III; nuptial pad absent; subarticular tubercles absent; large, rounded inner palmar tubercle distinctly separated from small, rounded outer palmar tubercle; webbing and lateral fringes on fingers absent. Tips of toes rounded, slightly swollen; relative toe length I $<$ II $<$ V $<$ III $<$ IV; subarticular tubercles absent; distinct longitudinal dermal ridges present under the $3^{\text {rd }}$ to $5^{\text {th }}$ toes, not interrupted; large, oval inner metatarsal tubercle 
present, outer metatarsal tubercle absent; toes webbing rudimentary; narrow lateral fringes present on all toes. Tibia $47 \%$ of snout-vent length; tibiotarsal articulation reaching to anterior corner of eye; heels slightly overlapping when thighs are appressed at right angles with respect to body.

Dorsal skin rough, with dense conical granules, tubercles and glandular folds; ventral skin smooth; sparse tiny tubercles present on surface of chest; pectoral gland and femoral gland oval; the size of pectoral glands almost equal to tips of fingers and femoral glands; femoral gland situated on posteroventral surface of thigh, closer to knee than to vent; supra-axillary glands raised. Ventrolateral glands distinctly visible, raised, forming an incomplete line.

Coloration of holotype in life. Dorsum greyish brown with small light orange granules, distinct darker brown markings scattered with irregular light orange and greyish white pigmentations. A dark brown inverted triangular pattern between the anterior corners of the eyes in connection with a dark brown W-shaped marking in the interorbital region, which is also connected to a W-shaped marking between the axillae. Tympanum dark brown. Small light orange granules present on dorsum of body and limbs; a dark brown blotch under the eye; transverse dark brown bars present on dorsal surface of limbs and digits; distinct dark brown patches with light yellowish green margin on flanks from groin to axilla; elbow and upper arms with distinct coppery orange coloration.

Ventral surface of throat, chest, and belly creamy white; presence of distinct nebulous greyish speckles present on throat, and distinct dark patches on chest and abdomen; ventral surface of limbs greyish purple, scattered with greyish white spots and small patches. Supra-axillary gland coppery orange; femoral, pectoral, and ventrolateral glands greyish white. Iris bicolored, amber on upper half and silver on lower half.

Coloration of holotype in preservative (Fig. 4A). Dorsum of body and limbs dark brown; transverse bars on limbs become more distinct; dark brown patterns, markings and spots on the back become indistinct, orange pigmentations become dark brown, greyish white pigmentations become dark grey. Ventral surface of limbs and surface of throat light brown, surface of abdomen greyish white, nebulous speckles on throat absent, dark patches on chest, abdomen and flanks become more distinct, light yellowish green margin of patches on flanks absent. Supra-axillary, femoral, pectoral, and ventrolateral glands greyish white.

Variation. Measurements and body proportions are listed in Table 4. Nonsexual characters of all the female paratypes (SYS a007744, 7745, 7746) match the overall characters of the holotype except that: the dorsum is greyish brown in the holotype SYS a007743 (vs. yellowish brown in the paratypes); the size of the pectoral glands are almost equal to the tips of the fingers and the femoral glands (vs. the size of the pectoral glands are larger than the tips of fingers and the femoral glands in the paratypes); the tibia-tarsal articulation reaches forward to the anterior corner of the eye in the holotype (vs. the tibia-tarsal articulation reaches forward to the middle of the eye in the paratypes SYS a007745, 7746); the ventral skin of the thighs smooth (vs. the ventral skin of the thighs rough with dense raised tubercles in the paratypes). 

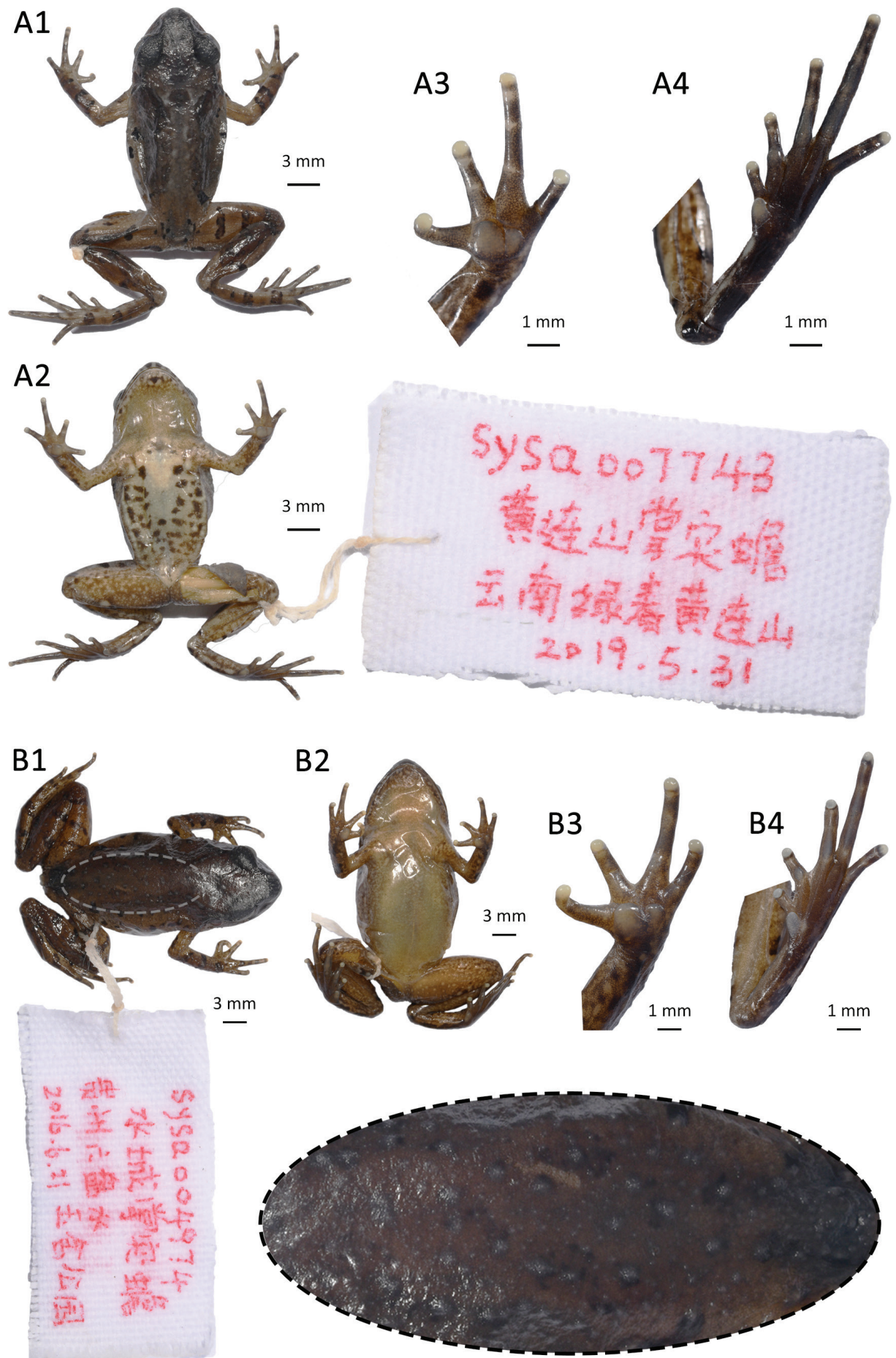

Figure 4. Morphological features in preserved specimens of A Leptobrachella aspera sp. nov., holotype SYS a007743 B Leptobrachella dorsospina sp. nov., holotype SYS a004974. Ellipse selected region showing the tiny spines on dorsal skin. 
Table 4. Measurements and body proportions of Leptobrachella aspera sp. nov.

\begin{tabular}{|c|c|c|c|c|}
\hline Voucher & SYS a 007743 & SYS a 007744 & SYS a 007745 & SYS a 007746 \\
\hline Sex & Male & Female & Female & Female \\
\hline SVL & 22.4 & 25.3 & 25.0 & 26.4 \\
\hline HDL & 8.1 & 9.5 & 9.5 & 9.6 \\
\hline HDW & 8.0 & 9.3 & 9.2 & 9.0 \\
\hline SNT & 3.7 & 3.8 & 3.8 & 3.4 \\
\hline IND & 2.5 & 2.3 & 2.7 & 2.7 \\
\hline IOD & 2.3 & 2.5 & 2.5 & 2.5 \\
\hline EYE & 2.9 & 3.2 & 3.2 & 3.1 \\
\hline TMP & 1.5 & 1.8 & 1.9 & 1.6 \\
\hline TEY & 0.7 & 1.0 & 1.0 & 0.8 \\
\hline ML & 5.9 & 7.0 & 6.6 & 6.3 \\
\hline LAHL & 11.2 & 13.5 & 12.7 & 12.6 \\
\hline PL & 10.1 & 11.7 & 10.2 & 11.1 \\
\hline TIB & 10.6 & 12.4 & 11.9 & 11.9 \\
\hline HLL & 34.4 & 41.5 & 40.4 & 39.1 \\
\hline HDL/SVL & 0.36 & 0.37 & 0.38 & 0.36 \\
\hline HDW/SVL & 0.36 & 0.37 & 0.37 & 0.34 \\
\hline HDW/HDL & 0.99 & 0.98 & 0.97 & 0.94 \\
\hline SNT/HDL & 0.16 & 0.15 & 0.15 & 0.13 \\
\hline IND/HDW & 0.31 & 0.25 & 0.29 & 0.30 \\
\hline IOD/HDW & 0.29 & 0.27 & 0.27 & 0.28 \\
\hline IND/IOD & 1.07 & 0.91 & 1.08 & 1.09 \\
\hline EYE/HDL & 0.36 & 0.34 & 0.34 & 0.32 \\
\hline TMP/EYE & 0.52 & 0.56 & 0.60 & 0.51 \\
\hline ML/SVL & 0.26 & 0.28 & 0.26 & 0.24 \\
\hline LAHL/SVL & 0.50 & 0.53 & 0.51 & 0.48 \\
\hline PL/SVL & 0.45 & 0.46 & 0.41 & 0.42 \\
\hline TIB/SVL & 0.47 & 0.49 & 0.48 & 0.45 \\
\hline HLL/SVL & 1.53 & 1.64 & 1.61 & 1.48 \\
\hline
\end{tabular}

Etymology. The specific epithet, aspera, is a Latin adjective which means rough, in reference to the dorsal skin texture of the new species. According to its type locality, we suggest its English common name as "Huanglianshan Leaf Litter Toad", and the Chinese name "Huang Lian Shan Zhang Tu Chan (黄连山掌突蟾)".

Distribution and habits. Currently, Leptobrachella aspera sp. nov. is known only from its type locality Huanglianshan Nature Reserve, near the border between China and Vietnam. The new species was found along a drainage ditch of a mountainous road. The road was surrounded by broad-leaved forest at an altitude ca. $1930 \mathrm{~m}$ and not close to any hillstreams. Males were not heard calling during the field survey from 31 May to 1 June 2019.

Leptobrachella dorsospina Wang, Lyu, Qi \& Wang, sp. nov. http://zoobank.org/B0EA8FA8-0193-43BF-AA93-6D010467CF84 Fig. 5

Type material. Holotype. SYS a004974, adult male, collected by Zhi-Tong Lyu and Run-Lin Li on 21 June 2016 from Yushe Forest Park $\left(26.47^{\circ} \mathrm{N}, 104.80^{\circ} \mathrm{E}\right.$; ca. $2100 \mathrm{~m}$ a.s.l.), Shuicheng District, Liupanshui City, Guizhou Province, China. 
Paratypes $(\mathrm{N}=6)$. An adult male, SYS a004977, and five adult females, SYS a004961/CIB116081, SYS a 004962, SYS a004973, 4975, 4976, collected by ZhiTong Lyu and Run-Lin Li on 20-21 June 2016 from the same locality as the holotype.

Diagnosis. (1) Small size (SVL 28.7-30.5 mm in two adult males, $32.1-39.8 \mathrm{~mm}$ in five adult females), (2) dorsal skin rough, with dense conical granules, tubercles, glandular folds and conical spines, (3) iris bicolored, light orange on upper half and silver on lower half, (4) tympanum distinctly discernible, distinct black supratympanic line present, (5) absence of webbing and lateral fringes on fingers, toes with rudimentary webbing and narrow lateral fringes both in males and females, (6) longitudinal ridges under toes interrupted at the articulations, (7) relative finger lengths II = IV $<$ I $<$ III, relative toe length I $<$ II $<$ V $<$ III $<$ IV, (8) heels slightly overlapping, tibia-tarsal articulation reaches forward to the posterior corners of eyes, (9) dorsum greyish brown to dark brown grounding, with distinct darker brown markings and scattered with irregular light greyish brown pigmentations and yellowish brown spots, (10) flanks with several enlarged dark patches positioned longitudinally in two rows, (11) ventral surface greyish white with black spots and orange pigmentations.

Comparison. Compared with the 26 known congeners of the genus Leptobrachella occurring south of the Kra Isthmus, $L$. dorsospina sp. nov. can be easily distinguished by the presence of supra-axillary and ventrolateral glands, from $L$. arayai, $L$. dringi, L. fritinniens, $L$. gracilis, $L$. hamidi, L. heteropus, L. kajangensis, L. kecil, L. marmorata, L. melanoleuca, L. maura, L. picta, L. platycephala, L. sabahmontana and L. sola, all of which are lacking the supra-axillary and ventrolateral glands; and by the significantly larger body size, SVL $28.7-30.5 \mathrm{~mm}$ in two adult male, $L$. dorsospina sp. nov. differs from the smaller L. baluensis (14.9-15.9 $\mathrm{mm}$ in males), L. brevicrus (17.1-17.8 mm in males), L. bondangensis (17.8 $\mathrm{mm}$ in male), L. fusca (16.3 $\mathrm{mm}$ in male), L. itiokai (15.2-16.7 $\mathrm{mm}$ in males), L. juliandringi (17.0-17.2 $\mathrm{mm}$ in males), L. mjobergi (15.7$19.0 \mathrm{~mm}$ in males), $L$. natunae (17.6 $\mathrm{mm}$ in one adult male), $L$. parva $(15.0-16.9 \mathrm{~mm}$ in males), L. palmata (14.4-16.8 $\mathrm{mm}$ in males), and L. serasanae (16.9 $\mathrm{mm}$ in female).

Leptobrachella dorsospina sp. nov. can be easily distinguished from Leptobrachella aspera sp. nov. by having distinctly larger body size, SVL $28.7-30.5 \mathrm{~mm}$ in males, $32.1-39.8 \mathrm{~mm}$ in females (vs. SVL $22.4 \mathrm{~mm}$ in male, 25.0-26.4 in females); conical spines on dorsal skin present (vs. absent); black spots on flanks in one row (vs. black spots on flanks in two rows); ventral skin greyish white with black spots and orange pigmentations (vs. ventral skin creamy white with distinct dark patches on chest and abdomen); longitudinal ridges under toes interrupted at the articulations (longitudinal ridges under toes not interrupted at the articulations).

For the remaining 56 members of the genus Leptobrachella, in having SVL 28.7$30.5 \mathrm{~mm}$ in two males, L. dorsospina sp. nov. differs from the larger L. eos (33.1$34.7 \mathrm{~mm}$ in males), L. nahangensis (40.8 $\mathrm{mm}$ in male), L. sungi $(48.3-52.7 \mathrm{~mm}$ in males), L. tamdil (32.3 $\mathrm{mm}$ in male), and L. zhangyapingi ( $45.8-52.5 \mathrm{~mm}$ in males); and from the smaller L. alpina (24.0-26.4 mm in males), L. applebyi $(19.6-22.3 \mathrm{~mm}$ in males), L. ardens (21.3-24.7 mm in males), L. bidoupensis (18.5-25.4 $\mathrm{mm}$ in males), L. crocea (22.2-27.3 $\mathrm{mm}$ in males), L. feii (21.5-22.8 $\mathrm{mm}$ in males), L. flaviglandulosa (23.0-27.0 $\mathrm{mm}$ in males), L. isos (23.7-27.9 $\mathrm{mm}$ in males), L. khasiorum (24.5- 


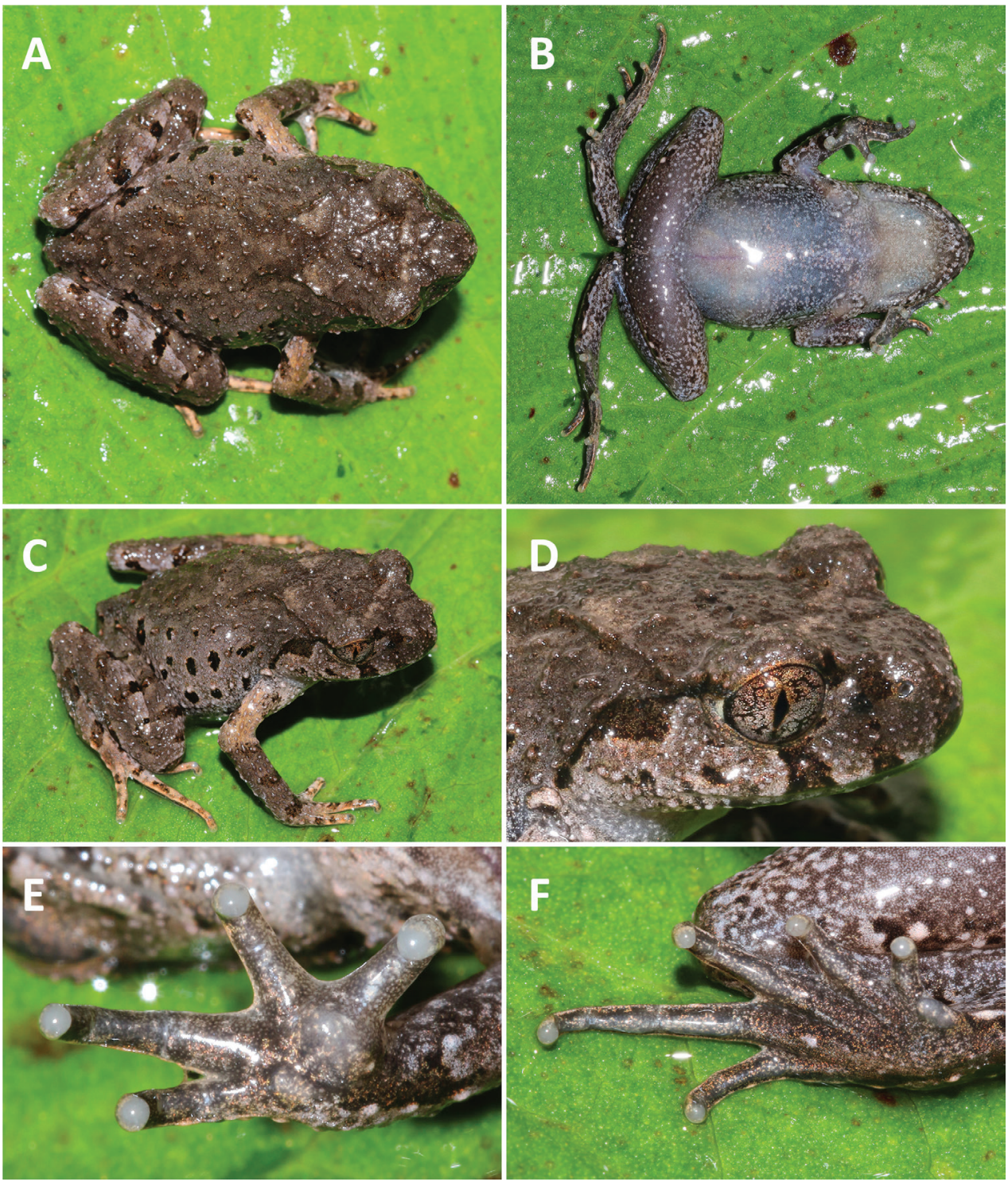

Figure 5. Morphological features in life. Leptobrachella dorsospina sp. nov., holotype SYS a004974.

$27.3 \mathrm{~mm}$ in males), L. laui (24.8-26.7 $\mathrm{mm}$ in males), L. maculosa (24.2-26.6 mm in males), L. mangshanensis (22.2-27.8 mm in males), L. melica (19.5-22.7 $\mathrm{mm}$ in males), L. niveimontis (22.5-23.6 $\mathrm{mm}$ in males), L. pallida (24.5-27.7 $\mathrm{mm}$ in males), L. petrops (23.6-27.6 mm in males), L. pluvialis (21.3-22.3 $\mathrm{mm}$ in males), L. puhoatensis (24.2-28.1 $\mathrm{mm}$ in males), L. purpura (25.0-27.5 $\mathrm{mm}$ in males), L. rowleyae (23.4$25.4 \mathrm{~mm}$ in males), L. tadungensis (23.3-28.2 $\mathrm{mm}$ in males), L. tengchongensis (23.9$26.0 \mathrm{~mm}$ in males), L. ventripunctata (25.5-28.0 $\mathrm{mm}$ in males), and L. yingjiangensis (25.7-27.6 $\mathrm{mm}$ in males). By having black spots on the flanks, L. dorsospina sp. nov. can be distinguished from L. aerea, L. botsfordi, L. firthi, and L. tuberosa, all of which 
lack black spots on the flanks. By having rough dorsal skin with conical spines, the new species can be distinguished from L. bijie, L. chishuiensis, L. liui, L. maoershanensis, L. pyrrhops, L. purpuraventra, L. suiyangensis, L. wuhuangmontis, L. wulingensis, and L. yunkaiensis (dorsal skin lacking spines); and from L. bourreti, L. fuliginosa, L. kalonensis, L. minima, L. oshanensis, and L. pelodytoides (dorsal skin smooth). By having narrow lateral fringes on the toes, the new species can be distinguished from L. lateralis, L. macrops, L. nyx, L. pyrrhops, L. namdongensis and L. neangi, all of which lack lateral fringes on the toes. The new species can be separated from the remaining L. nokrekensis by having greyish white ventral coloration with black patches and orange pigmentations (vs. creamy white), and having dense short glandular folds on the dorsal surface (vs. only a few glandular folds on the dorsal surface).

Description of holotype. Adult male. Body size rather small, SVL $30.5 \mathrm{~mm}$. Head length slightly larger than head width, HDW/HDL 0.99 ; snout slightly protruding, projecting slightly beyond margin of the lower jaw; nostril closer to snout than eye; canthus rostralis gently rounded; loreal region slightly concave; interorbital space flat, internarial distance smaller than interorbital distance, IND/IOD 0.91; pineal ocellus absent; vertical pupil; snout length larger than eye diameter, SNT/EYE 1.29; tympanum distinct, rounded, and slightly concave, diameter smaller than that of the eye and larger than tympanum-eye distance, TMP/EYE 0.43 and TEY/TMP 0.50; upper margin of tympanum in contact with supratympanic ridge; distinct black supratympanic line present; vomerine teeth absent; vocal sac openings slit-like, paired, located posterolaterally on floor of mouth in close proximity to the margins of the mandible; tongue deeply notched posteriorly; supratympanic ridge distinct, extending from posterior corner of eye to supra-axillary gland.

Tips of fingers rounded, slightly swollen; relative finger lengths II $=$ IV $<$ I $<$ III; nuptial pad absent; subarticular tubercles absent; large, rounded inner palmar tubercle distinctly separated from small, rounded outer palmar tubercle; absence of webbing and lateral fringes on fingers. Tips of toes rounded, slightly swollen; relative toe length I $<$ II $<$ V $<$ III $<$ IV; subarticular tubercles absent; distinct longitudinal dermal ridges present under the $3^{\text {rd }}$ to $5^{\text {th }}$ toes, interrupted; large, oval inner metatarsal tubercle present, outer metatarsal tubercle absent; toes webbing rudimentary; narrow lateral fringes present on all toes. Tibia $44 \%$ of snout-vent length; tibiotarsal articulation reaches to posterior corner of eye; heels slightly overlapping when thighs are appressed at right angles with respect to body.

Dorsal skin rough, with dense conical granules, tubercles, glandular folds and conical spines; ventral skin smooth; pectoral gland and femoral gland oval; the size of pectoral glands almost equal to tips of fingers and femoral glands; femoral gland situated on posteroventral surface of thigh, closer to knee than to vent; supra-axillary glands raised. Ventrolateral glands distinctly visible, raised, forming an incomplete line.

Coloration of holotype in life. Dorsum greyish brown with distinct darker brown markings on sides and scattered with irregular light greyish brown pigmentations and yellowish brown spots. An indistinct, darker brown inverted triangular pattern between anterior corners of the eyes, connected to an indistinct dark brown W-shaped marking between the axillae. Dense translucent spines present on dorsal skin of body 
and limbs. Upper 2/3 of the tympanum dark brown, lower $1 / 3$ light orange, scattered with tiny coppery orange spots. Small greyish white and light brown granules present on the dorsum of the body and limbs; a dark brown vertical bar under the eye; transverse dark brown bars on the dorsal surface of the limbs and digits; distinct dark brown patches on the flanks, from groin to axilla; elbow and upper arms with distinct light orange coloration.

Ventral surface of throat, chest, and belly greyish white; throat with light brown speckles, chest, and abdomen with distinct dark patches; ventral surface of limbs dark grey, scattered with greyish white spots and small patches. Supra-axillary gland light orange; femoral, pectoral, and ventrolateral glands greyish white. Iris bicolored, light orange on upper half and silver on lower half.

Coloration of holotype in preservative (Fig. 4B). Dorsum of body and limbs dark brown; transverse bars on limbs, dark brown patterns, markings, and spots on back become indistinct, light greyish brown pigmentations and yellowish spots absent. Translucent spines on dorsal skin of body and limbs become grey. Ventral surface of limbs and surface of throat light brown, surface of abdomen greyish white, dark patches on chest, abdomen and flanks become more distinct. Supra-axillary, femoral, pectoral, and ventrolateral glands greyish white.

Variations. Measurements and body proportions are listed in Table 5. All the female paratypes match the overall characters of the holotype except that: the dorsum

Table 5. Measurements, and body proportions of Leptobrachella dorsospina sp. nov.

\begin{tabular}{lccccccc}
\hline \multicolumn{1}{c}{ Voucher } & SYS a004977 & SYS a004974 & SYS a004961 & SYS a004962 & SYS a004973 & SYS a004975 & SYS a004976 \\
\hline Sex & Male & Male & Female & Female & Female & Female & Female \\
SVL & 28.7 & 30.5 & 36.1 & 37.3 & 39.8 & 32.1 & 33.8 \\
HDL & 10.3 & 10.8 & 12.8 & 12.4 & 12.9 & 11.3 & 12.0 \\
HDW & 10.6 & 10.7 & 11.9 & 12.9 & 13.2 & 11.8 & 12.2 \\
SNT & 4.5 & 4.2 & 5.1 & 5.4 & 5.8 & 5.0 & 4.8 \\
IND & 3.1 & 3.2 & 3.6 & 3.9 & 4.0 & 3.7 & 3.4 \\
IOD & 3.4 & 2.9 & 3.5 & 3.4 & 3.3 & 3.0 & 2.9 \\
EYE & 3.5 & 3.7 & 3.9 & 3.7 & 4.3 & 4.2 & 3.8 \\
TMP & 1.7 & 1.6 & 2.3 & 2.3 & 2.6 & 2.1 & 2.1 \\
TEY & 1.1 & 0.8 & 1.3 & 1.4 & 1.5 & 1.2 & 1.1 \\
ML & 7.4 & 7.3 & 8.8 & 7.7 & 9.1 & 7.8 & 7.6 \\
LAHL & 14.1 & 14.2 & 17.1 & 16.8 & 17.5 & 16.2 & 15.9 \\
PL & 12.1 & 12.8 & 14.9 & 14.5 & 15.5 & 13.9 & 13.6 \\
TIB & 13.5 & 13.4 & 15.5 & 16.3 & 16.6 & 14.9 & 14.5 \\
HLL & 41.7 & 42.7 & 49.1 & 49.9 & 52.9 & 46.8 & 48.0 \\
HDL/SVL & 0.36 & 0.35 & 0.35 & 0.33 & 0.32 & 0.35 & 0.36 \\
HDW/SVL & 0.37 & 0.35 & 0.33 & 0.35 & 0.33 & 0.37 & 0.36 \\
HDW/HDL & 1.03 & 0.99 & 0.93 & 1.04 & 1.02 & 1.04 & 1.02 \\
SNT/HDL & 0.44 & 0.39 & 0.40 & 0.44 & 0.45 & 0.44 & 0.40 \\
IND/HDW & 0.29 & 0.30 & 0.30 & 0.30 & 0.30 & 0.31 & 0.28 \\
IOD/HDW & 0.32 & 0.27 & 0.29 & 0.26 & 0.25 & 0.25 & 0.24 \\
EYE/HDL & 0.34 & 0.34 & 0.30 & 0.30 & 0.33 & 0.37 & 0.32 \\
TMP/EYE & 0.49 & 0.43 & 0.59 & 0.62 & 0.60 & 0.50 & 0.55 \\
ML/SVL & 0.26 & 0.24 & 0.24 & 0.21 & 0.23 & 0.24 & 0.22 \\
LAHL/SVL & 0.49 & 0.47 & 0.47 & 0.45 & 0.44 & 0.50 & 0.47 \\
PL/SVL & 0.42 & 0.42 & 0.41 & 0.39 & 0.39 & 0.43 & 0.40 \\
TIB/SVL & 0.47 & 0.44 & 0.43 & 0.44 & 0.42 & 0.46 & 0.43 \\
HLL/SVL & 1.45 & 1.40 & 1.36 & 1.34 & 1.33 & 1.46 & 1.42 \\
\hline
\end{tabular}


is greyish brown in the holotype SYS a004974 (vs. dark brown in the paratypes SYS a004961, 4962), and black spots on the ventral skin are more dense and distinct in the paratypes SYS a004961, 4962.

Etymology. The specific epithet, dorsospina, is in reference to the conical spines on the dorsal surface of body in the new species. According to its type locality, we suggest its English common name as "Shuicheng Leaf Litter Toad", and the Chinese name “Shui Cheng Zhang Tu Chan (水城掌突蟾)".

Distribution and habits. Currently, Leptobrachella dorsospina sp. nov. is known only from its type locality, Yushe Forest Park, which is near the border between Guizhou and Yunnan. The new species was found on the surface of fallen leaves by the clear-water rocky hill-stream in well-preserved montane evergreen broadleaf forest (ca. $2100 \mathrm{~m}$ a.s.l.). Males were not heard calling.

\section{Discussion}

In the phylogenetic tree, the Leptobrachella pelodytoides (voucher number: MVZ 223642) sample from Tam Dao, northern Vietnam is clustered together with the topotypic $L$. ventripunctata (voucher number: SYS a001768) sample from Xishuangbanna, Yunnan, China, with a genetic divergence of only $1.5 \%$ (Fig. 2, Suppl. material 1: Table S1), which is of a intraspecific level. In addition, the type locality of $L$. pelodytoides is Thao [= Thamo], Kayah State, Myanmar, which is geographically distant from northern Vietnam with a distance over $900 \mathrm{~km}$. Considering the above, we recommend that the specimen MVZ 223642 be reappraised as L. ventripunctata.

Yunnan and Guizhou are both largely within the species-rich Dian freshwater zoogeographical dominion (Huang et al 2020). Spanning the Indo-Burma Hotspot and the Mountains of Southwest China Hotspot (Tordoff et al. 2012), Yunnan Province has for long been considered as one of the most biodiverse regions in China and its flora and fauna have attracted much attention. However, Guizhou Province, which also shares the Yunnan-Guizhou Plateau, remains relatively neglected; knowledge of biodiversity levels and patterns are seriously lacking. In recent years, large numbers of discoveries have been made from Guizhou, dramatically raising the number of frog species known from the region (Zhang et al. 2017; Li et al. 2018a, b, 2019a, b, 2020a; Lyu et al. 2019; Wang et al. 2019; Luo et al. 2020; Wei et al. 2020). Further comprehensive surveys are urgently needed to determine the true diversity of the amphibians of Guizhou Province.

\section{Acknowledgements}

We are grateful to Prof. Tao Thien Nguyen, Prof. Annemarie Ohler, and an anonymous reviewer for providing constructive comments that greatly improved the quality of the manuscript. We would like to thank Yu-Long Li, Run-Lin Li and Hong-Hui Chen for their help in the field work, and Yao Li for her help in the lab work. 


\section{References}

Anderson J (1871) A list of the reptilian accession to the Indian Museum, Calcutta from 1865 to 1870 , with a description of some new species. Journal of the Asiatic Society of Bengal 40: 12-39.

Boulenger GA (1893) Concluding report on the reptiles and batrachians obtained in Burma by Signor L. Fea dealing with the collection made in Pegu and the Karin Hills in 1887-88. Annali del Museo Civico di Storia Naturale di Genova 13: 304-347.

Boulenger GA (1900) Descriptions of new batrachians and reptiles from the Larut Hills, Perak. Annals and Magazine of Natural History 6: 186-194. https://doi. org/10.1080/00222930008678356

Castresana J (2000) Selection of conserved blocks from multiple alignments for their use in phylogenetic analysis. Molecular Biology and Evolution 17: 540-552. https://doi. org/10.1093/oxfordjournals.molbev.a026334

Chen JM, Zhou WW, Poyarkov NA, Stuart BL, Brown RM, Lathrop A, Wang YY, Yuan ZY, Jiang K, Hou M, Chen HM, Suwannapoom C, Nguyen SN, Duong TV, Papenfuss TJ, Murphy RW, Zhang YP, Che J (2017) A novel multilocus phylogenetic estimation reveals unrecognized diversity in Asian horned toads, genus Megophrys sensu lato (Anura: Megophryidae). Molecular Phylogenetics and Evolution 106: 28-43. https://doi. org/10.1016/j.ympev.2016.09.004

Chen JM, Poyarkov NJ, Suwannapoom C, Lathrop A, Wu YH, Zhou WW, Yuan ZY, Jin JQ, Chen HM, Liu HQ, Nguyen TQ, Nguyen SN, Duong TV, Eto K, Nishikawa K, Matsui M, Orlov NL, Stuart BL, Brown RM, Rowley J, Murphy RW, Wang YY, Che J (2018) Large-scale phylogenetic analyses provide insights into unrecognized diversity and historical biogeography of Asian leaf-litter frogs, genus Leptolalax (Anura: Megophryidae). Molecular Phylogenetics and Evolution 124: 162-171. https://doi.org/10.1016/j. ympev.2018.02.020

Chen WC, Liao X, Zhou SC, Mo YM (2019) A new species of Leptobrachella (Anura: Megophryidae) from southern Guangxi, China. Zootaxa 4563: 67-82. https://doi.org/10.11646/ zootaxa.4563.1.3

Chen JM, Xu K, Poyarkov NA, Wang K, Yuan ZY, Hou M, Suwannapoom C, Wang J, Che J (2020) How little is known about "the little brown frogs": description of three new species of the genus Leptobrachella (Anura: Megophryidae) from Yunnan Province, China. Zoological Research 41: 1-22. https://doi.org/10.24272/j.issn.2095-8137.2020.036

Darriba D, Taboada GL, Doallo R, Posada D (2012) jModelTest 2: more models, new heuristics and parallel computing. Nature methods 9: 772-772. https://doi.org/10.1038/ nmeth.2109

Das I, Tron RKL, Rangad D, Hooroo RN (2010) A new species of Leptolalax (Anura: Megophryidae) from the sacred groves of Mawphlang, Meghalaya, north-eastern India. Zootaxa 2339: 44-56. https://doi.org/10.11646/zootaxa.2339.1.2

Dehling JM (2012a) Eine neue Art der Gattung Leptolalax (Anura: Megophryidae) vom Gunung Benom, Westmalaysia/A new species of the genus Leptolalax (Anura: Megophryidae) from Gunung Benom, Peninsular Malaysia. Sauria 34: 9-21. 
Dehling JM (2012b) Redescription of Leptolalax gracilis (Günther, 1872) from Borneo and taxonomic status of two populations of Leptolalax (Anura: Megophryidae) from Peninsular Malaysia. Zootaxa 3328: 20-34. https://doi.org/10.11646/zootaxa.3328.1.2

Dehling JM, Matsui M (2013) A new species of Leptolalax (Anura: Megophryidae) from Gunung Mulu National Park, Sarawak, East Malaysia (Borneo). Zootaxa 3670(1): 33-44.

Delorme M, Dubois A, Grosjean S, Ohler A (2006) Une nouvelle ergotaxinomie des Megophryidae (Amphibia, Anura). Alytes 24: 6-21.

Dring J (1983) Frogs of the genus Leptobrachella (Pelobatidae). Amphibia-Reptillia 4: 89-102. https://doi.org/10.1163/156853883X00012

Dubois A (1983) Note préliminaire sur le genre Leptolalax Dubois, 1980 (Amphibiens, Anoures), avec diagnose d'une espèce novelle du Vietnam. Alytes 2: 147-153.

Dubois A, Grosjean S, Ohler A, Adler K, Zhao EM (2010) The nomenclatural status of some generic nomina of Megophryidae (Amphibia, Anura). Zootaxa 2493: 66-68. https://doi. org/10.11646/zootaxa.2493.1.6

Duong TV, Do DT, Ngo CD, Nguyen TQ, Poyarkov Jr NA (2018) A new species of the genus Leptolalax (Anura: Megophryidae) from southern Vietnam. Zoological Research 39: 181-196.

Eto K, Matsui M, Nishikawa K (2015) Description of a new species of the genus Leptobrachella (Amphibia, Anura, Megophryidae) from Borneo. Current Herpetology 34(2): 128-139. https://doi.org/10.5358/hsj.34.128

Eto K, Matsui M, Nishikawa K (2016) A new highland species of dwarf litter frog genus Leptobrachella (Amphibia, Anura, Megophryidae) from Sarawak. Raffles Bulletin of Zoology 64: 194-203.

Eto K, Matsui M, Hamidy A, Munir M, Iskandar DT (2018) Two new species of the genus Leptobrachella (Amphibia: Anura: Megophryidae) from Kalimantan, Indonesia. Current Herpetology 37(2): 95-105. https://doi.org/10.5358/hsj.37.95

Fei L, Hu SQ, Ye CY, Huang YZ (2009) Fauna Sinica. Amphibia Vol. 2 Anura. Science Press, Beijing, 957 pp. [In Chinese]

Fei L, Ye CY, Jiang JP (2012) Colored atlas of Chinese amphibians and their distributions. Sichuan Publishing House of Science \& Technology, Chengdu, 619 pp. [In Chinese]

Frost DR (2020) Amphibian Species of the World: an Online Reference. Version 6.0. Electronic Database. American Museum of Natural History, New York. http://research.amnh. org/herpetology/amphibia/index.html [accessed 28 June 2020]

Grismer LL, Grismer JL, Youmans TM (2004) A new species of Leptolalax (Anura: Megophryidae) from Pulau Tioman, West Malaysia. Asiatic Herpetological Research 10: 8-11.

Günther A (1872) On the reptiles and amphibians of Borneo. Proceedings of the Scientific Meetings of the Zoological Society of London 1872: 586-600.

Günther A (1985) The reptiles and batrachians of the Natuna Islands. Novitates Zoologicae 2: 499-502.

Huang C, Ebach MC, Ahyong ST (2020) Bioregionalisation of the freshwater zoogeographical areas of mainland China. Zootaxa 4742(2): 271-298. https://doi.org/10.11646/ zootaxa.4742.2.3

Humtsoe LN, Bordoloi S, Ohler A, Dubois A (2008) Rediscovery of a long known species, Ixalus lateralis Anderson, 1871. Zootaxa 1921: 24-34. https://doi.org/10.11646/zootaxa.1921.1.2 
Hou YM, Zhang MF, Hu F, Li SY, Shi SC, Chen J, Mo XY, Wang B (2018) A new species of the genus Leptolalax (Anura, Megophryidae) from Hunan, China. Zootaxa 4444(3): 247-266. https://doi.org/10.11646/zootaxa.4444.3.2

Hoang CV, Nguyen TT, Luu VQ, Nguyen TQ, Jiang JP (2019) A new species of Leptobrachella Smith 1925 (Anura: Megophryidae) from Thanh Hoa Province, Vietnam. Raffles Bulletin of Zoology 67: 536-556.

Inger RF, Lakim M, Biun A, Yambun P (1997) A new species of Leptolalax (Anura: Megophryidae) from Borneo. Asiatic Herpetological Research 7: 48-50. https://doi.org/10.5962/bhl. part. 18855

Inger RF, Orlov N, Darevsky I (1999) Frogs of Vietnam: a report on new collections. Fieldiana Zoology 92: 1-46.

Inger RF, Stuebing RB, Tan FL (1995) New species and new records of anurans from Borneo. Raffles Bulletin of Zoology 43: 115-132.

Jiang K, Yan F, Suwannapoom C, Chomdej S, Che J (2013) A new species of the genus Leptolalax (Anura: Megophryidae) from northern Thailand. Asian Herpetological Research 4(2): 100-108. https://doi.org/10.3724/SP.J.1245.2013.00100

Lathrop A, Murphy RW, Orlov N, Ho CT (1998) Two new species of Leptolalax (Anura: Megophryidae) from northern Vietnam. Amphibia-Reptilia 19: 253-267. https://doi. org/10.1163/156853898X00160

Luo T, Xiao N, Gao K, Zhou J (2020) A new species of Leptobrachella (Anura, Megophryidae) from Guizhou Province, China. ZooKeys 923: 115-140. https://doi.org/10.3897/zookeys.923.47172

Li SZ, Xu N, Lv JC, Jiang JP, Wei G, Wang B (2018a) A new species of the odorous frog genus Odorrana (Amphibia, Anura, Ranidae) from southwestern China. PeerJ 6(e5695): 1-28. https://doi.org/10.7717/peerj.5695

Li SZ, Xu N, Liu J, Jiang JP, Wei G, Wang B (2018b) A new species of the Asian Toad genus Megophrys sensu lato (Amphibia: Anura: Megophryidae) from Guizhou Province, China. Asian Herpetological Research 9: 224-239. https://doi.org/10.16373/j.cnki.ahr.180072

Li SZ, Wei G, Xu N, Cui JG, Fei L, Jiang JP, Liu J, Wang B (2019a) A new species of the Asian music frog genus Nidirana (Amphibia, Anura, Ranidae) from Southwestern China. PeerJ 7: e7157. https://doi.org/10.7717/peerj.7157

Li SZ, Zhang MH, Xu N, Lv JC, Jiang JP, Liu J, Wei G, Wang B (2019b) A new species of the genus Microhyla (Amphibia: Anura: Microhylidae) from Guizhou Province, China. Zootaxa 4624: 551-575. https://doi.org/10.11646/zootaxa.4624.4.7

Li SZ, Liu J, Wei G, Wang B (2020a) A new species of the Asian leaf litter toad genus Leptobrachella (Amphibia, Anura, Megophryidae) from southwest China. ZooKeys 943: 91118. https://doi.org/10.3897/zookeys.943.51572

Li Y, Zhang DD, Lyu ZT, Wang J, Li YL, Liu ZY, Chen HH, Rao DQ, Jin ZF, Zhang CY, Wang YY (2020b) Review of the genus Brachytarsophrys (Anura: Megophryidae), with revalidation of Brachytarsophrys platyparietus and description of a new species from China. Zoological Research 41: 105-122. https://doi.org/10.24272/j.issn.20958137.2020.033

Liu ZY, Chen GL, Zhu TQ, Zeng ZC, Lyu ZT, Wang J, Messenger K, Greenberg AJ, Guo ZX, Yang ZH, Shi SH, Wang YY (2018) Prevalence of cryptic species in morphologically 
uniform taxa - Fast speciation and evolutionary radiation in Asian frogs. Molecular Phylogenetics and Evolution 127: 723-731. https://doi.org/10.1016/j.ympev.2018.06.020

Lyu ZT, Zeng ZC, Wan H, Yang JH, Li YL, Pang H, Wang YY (2019b) A new species of Amolops (Anura: Ranidae) from China, with taxonomic comments on A. liangshanensis and Chinese populations of $A$. marmoratus. Zootaxa 4609: 247-268. https://doi.org/10.11646/ zootaxa.4609.2.3

Malkmus R (1992) Leptolalax pictus sp.n. (Anura: Pelobatidae) vom Mount Kinabalu/NordBorneo. Sauria 14: 3-6.

Mahony S, Foley NM, Biju S, Teeling EC (2017) Evolutionary history of the Asian Horned Frogs (Megophryinae): integrative approaches to timetree dating in the absence of a fossil record. Molecular Biology and Evolution 34(3): 744-771. https://doi.org/10.1093/molbev/msw267

Matsui M (1997) Call characteristics of Malaysian Leptolalax with a description of two new species (Anura: Pelobatidae). Copeia 1997(1): 158-165. https://doi.org/10.2307/1447851

Matsui M (2006) Three new species of Leptolalax from Thailand (Amphibia, Anura, Megophryidae). Zoological Science 23(9): 821-830. https://doi.org/10.2108/zsj.23.821

Matsui M, Dehling JM (2012) Notes on an enigmatic Bornean megophryid, Leptolalax dringi Dubois, 1987 (Amphibia: Anura). Zootaxa 3317: 49-58. https://doi.org/10.11646/ zootaxa.3317.1.4

Matsui M, Belabut DM, Ahmad N, Yong HS (2009) A new species of Leptolalax (Amphibia, Anura, Megophryidae) from Peninsular Malaysia. Zoological Science 26(3): 243-247. https://doi.org/10.2108/zsj.26.243

Matsui M, Nishikawa K, Yambun P (2014a) A new Leptolalax from the mountains of Sabah, Borneo (Amphibia, Anura, Megophryidae). Zootaxa 3753(3): 440-452. https://doi. org/10.11646/zootaxa.3753.5.3

Matsui M, Zainudin R, Nishikawa K (2014b) A New Species of Leptolalax from Sarawak, Western Borneo (Anura: Megophryidae). Zoological Science 31(11): 773-779. https:// doi.org/10.2108/zs140137

Mathew R, Sen N (2010 [2009]) Description of a new species of Leptobrachium Tschudi, 1838 (Amphibia: Anura: Megophryidae) from Meghalaya, India. Records of the Zoological Survey of India 109: 91-108.

Nguyen LT, Poyarkov Jr NA, Le DT, Vo BD, Ninh HT, Duong TV, Murphy RW, Sang NV (2018) A new species of Leptolalax (Anura: Megophryidae) from Son Tra Peninsula, central Vietnam. Zootaxa 4388: 1-21. https://doi.org/10.11646/zootaxa.4388.1.1

Ohler A, Marquis O, Swan S, Grosjean S (2000) Amphibian biodiversity of Hoang Lien Nature Reserve (Lao Cai Province, northern Vietnam) with description of two new species. Herpetozoa 13(1/2): 71-87.

Ohler A, Wollenberg KC, Grosjean S, Hendrix R, Vences M, Ziegler T, Dubois A (2011) Sorting out Lalos: description of new species and additional taxonomic data on megophryid frogs from northern Indochina (genus Leptolalax, Megophryidae, Anura). Zootaxa 3147: 1-83. https://doi.org/10.11646/zootaxa.3147.1.1

Poyarkov NJ, Rowley JJ, Gogoleva SI, Vassilieva AB, Galoyan EA, Orlov NL (2015) A new species of Leptolalax (Anura: Megophryidae) from the western Langbian Plateau, southern Vietnam. Zootaxa 3931(2): 221-252. https://doi.org/10.11646/zootaxa.3931.2.3 
Qian TY, Xia X, Cao Y, Xiao NW, Yang DD (2020) A new species of the genus Leptobrachella (Anura: Megophryidae) Smith, 1925 from Wuling Mountains in Hunan Province, China. Zootaxa 4816(4): 491-526. https://doi.org/10.11646/zootaxa.4816.4.4

Ronquist F, Teslenko M, Van Der Mark P, Ayres DL, Darling A, Höhna S, Larget B, Liu L, Suchard MA, Huelsenbeck JP (2012) MrBayes 3.2: efficient Bayesian phylogenetic inference and model choicem across a large model space. Systematic Biology 61: 539-542. https://doi.org/10.1093/sysbio/sys029

Rowley JJ, Cao TT (2009) A new species of Leptolalax (Anura: Megophryidae) from central Vietnam. Zootaxa 2198: 51-60. https://doi.org/10.11646/zootaxa.2198.1.5

Rowley JJ, Hoang DH, Le TTD, Dau QV, Cao TT (2010a) A new species of Leptolalax (Anura: Megophryidae) from Vietnam and further information on Leptolalax tuberosus. Zootaxa 2660: 33-45.

Rowley JJ, Stuart BL, Neang T, Emmett DA (2010b) A new species of Leptolalax (Anura: Megophryidae) from northeastern Cambodia. Zootaxa 2567: 57-68. https://doi. org/10.11646/zootaxa.2567.1.3

Rowley JJ, Stuart BL, Richards SJ, Phimmachak S, Sivongxay N (2010c) A new species of Leptolalax (Anura: Megophryidae) from Laos. Zootaxa 2681: 35-46. https://doi.org/10.11646/ zootaxa.2681.1.3

Rowley JJ, Le DTT, Tran DTA, Hoang DH (2011) A new species of Leptobrachella (Anura: Megophryidae) from southern Vietnam. Zootaxa 2796: 15-28. https://doi.org/10.11646/ zootaxa.2796.1.2

Rowley JJ, Hoang HD, Dau VQ, Le TTD, Cao TT (2012) A new species of Leptolalax (Anura: Megophryidae) from central Vietnam. Zootaxa 3321: 56-68. https://doi.org/10.11646/ zootaxa.3321.1.4

Rowley JJ, Dau VQ, Nguyen TT (2013) A new species of Leptolalax (Anura: Megophryidae) from the highest mountain in Indochina. Zootaxa 3737(4): 415-428. https://doi. org/10.11646/zootaxa.3737.4.5

Rowley JJ, Stuart BL, Neang T, Hoang HD, Dau VQ, Nguyen TT, Emmett DA (2015a) A new species of Leptolalax (Anura: Megophryidae) from Vietnam and Cambodia. Zootaxa 4039: 401-417. https://doi.org/10.11646/zootaxa.4039.3.1

Rowley JJL, Tran DTA, Frankham GJ, Dekker AH, Le DTT, Nguyen TQ, Dau VQ, Hoang HD (2015b) Undiagnosed Cryptic Diversity in Small, Microendemic Frogs (Leptolalax) from the Central Highlands of Vietnam. PLoS ONE 10(5): e0128382. https://doi. org/10.1371/journal.pone.0128382

Rowley JJ, Tran DTA, Le DTT, Dau VQ, Peloso PLV, Nguyen TQ, Hoang HD, Nguyen TT, Ziegler T (2016) Five new, microendemic Asian Leaf-litter Frogs (Leptolalax) from the southern Annamite mountains, Vietnam. Zootaxa 4085: 63-102. https://doi. org/10.11646/zootaxa.4085.1.3

Rowley JJ, Dau VQ, Hoang HD, Le DTT, Cutajar TP, Nguyen TT (2017a) A new species of Leptolalax (Anura: Megophryidae) from northern Vietnam. Zootaxa 4243: 544-564. https://doi.org/10.11646/zootaxa.4243.3.7

Rowley JJ, Dau VQ, Cao TT (2017b) A new species of Leptolalax (Anura: Megophryidae) from Vietnam. Zootaxa 4273(1): 61-79. https://doi.org/10.11646/zootaxa.4273.1.5 
Sengupta S, Sailo S, Lalremsanga HT, Das A, Das I (2010) A new species of Leptolalax (Anura: Megophryidae) from Mizoram, north-eastern India. Zootaxa 2406: 56-68. https://doi. org/10.11646/zootaxa.2406.1.3

Silvestro D, Michalak I (2012) raxmlGUI: a graphical front-end for RAxML. Organisms Diversity \& Evolution 12: 335-337. https://doi.org/10.1007/s13127-011-0056-0

Simon C, Frati F, Beckenbach A, Crespi B, Liu H, Flook P (1994) Evolution, weighting, and phylogenetic utility of mitochondrial gene sequences and a compilation of conserved polymerase chain reaction primers. Annals of the Entomological Society of America 87: 651701. https://doi.org/10.1093/aesa/87.6.651

Sung YH, Yang JH, Wang YY (2014) A new species of Leptolalax (Anura: Megophryidae) from southern China. Asian Herpetological Research 5(2): 80-90. https://doi.org/10.3724/ SP.J.1245.2014.00080

Stuart BL, Rowley JJL (2020) A new Leptobrachella (Anura: Megophryidae) from the Cardamom Mountains of Cambodia. Zootaxa 4834(4): 556-572. https://doi.org/10.11646/ zootaxa.4834.4.4

Taylor EH (1962) The amphibian fauna of Thailand. University of Kansas Science Bulletin 43: 265-599. https://doi.org/10.5962/bhl.part.13347

Tamura K, Stecher G, Peterson D, Filipski A, Kumar S (2013) MEGA6: Molecular Evolutionary Genetics Analysis version 6.0. Molecular Biology and Evolution 30: 2725-2729. https://doi.org/10.1093/molbev/mst197

Thompson JD, Gibson TJ, Plewniak F, Jeanmougin F, Higgins DG (1997) The CLUSTAL_X windows interface: flexible strategies for multiple sequence alignment aided by quality analysis tools. Nucleic Acids Research 25: 4876-4882. https://doi.org/10.1093/ nar/25.24.4876

Wang J, Yang JH, Li Y, Lyu ZT, Zeng ZC, Liu ZY, Ye YH, Wang YY (2018) Morphology and molecular genetics reveal two new Leptobrachella species in southern China (Anura, Megophryidae). ZooKeys 776: 105-137. https://doi.org/10.3897/zookeys.776.22925

Wang J, Li YL, Li Y, Chen HH, Zeng YJ, Shen JM, Wang YY (2019) Morphology, molecular genetics, and acoustics reveal two new species of the genus Leptobrachella from northwestern Guizhou Province, China (Anura, Megophryidae). ZooKeys 848: 119-154. https:// doi.org/10.3897/zookeys.848.29181

Wei G, Li SZ, Liu J, Cheng YL, Xu N, Wang B (2020) A new species of the Music frog Nidirana (Anura, Ranidae) from Guizhou Province, China. ZooKeys 904: 63-87. https:// doi.org/10.3897/zookeys.904.39161

Yang JH, Wang YY, Chen GL, Rao DQ (2016) A new species of the genus Leptolalax (Anura: Megophryidae) from Mt. Gaoligongshan of western Yunnan Province, China. Zootaxa 4088: 379-394. https://doi.org/10.11646/zootaxa.4088.3.4

Yang JH, Zeng ZC, Wang YY (2018) Description of two new sympatric species of the genus Leptolalax (Anura: Megophryidae) from western Yunnan of China. PeerJ 6(e4586): 1-32. https://doi.org/10.7717/peerj.4586

Yuan ZY, Sun RD, Chen JM, Rowley JJ, Wu ZJ, Hou SB, Wang SN, Che J (2017) A new species of the genus Leptolalax (Anura: Megophryidae) from Guangxi, China. Zootaxa 4300: 551-570. https://doi.org/10.11646/zootaxa.4300.4.5 
Zhang Y, Li G, Xiao N, Li J, Pan T, Wang H, Zhang B, Zhou J (2017) A new species of the genus Xenophrys (Amphibia: Anura: Megophryicae) from Libo County, Guizhou, China. Asian Herpetological Research 8: 75-85.

\section{Appendix I}

\section{Specimens examined}

Leptobrachella alpina (n = 6): China: Yunnan Province: Jingdong County: Mt. Wuliang: CIB 24353 (holotype), CIB 24354; SYS a 003927.

Leptobrachella bijie (n = 8): China: Guizhou: Bijie City: SYS a007313-7320.

Leptobrachella laui (n = 26): China: Hong Kong: SYS a002057 (holotype), SYS a002058; China: Guangdong Province: Shenzhen City: SYSa 001505-1507, 1515-1521, 3471-3472, 5644-5645.

Leptobrachella liui (n = 18): China: Fujian Province: Mt. Wuyi: CIB 24355 (holotype), CIB 24356, SYS a001571-1578, 1595-1599, 2478-2479, 5925-5826.

Leptobrachella mangshanensis $(\mathbf{n}=5)$ : China: Guangdong: Nanling Nature Reserve: SYS a002827-2830, 5754.

Leptobrachella purpuraventra ( $\mathbf{n}=15)$ : China: Guizhou: Bijie City: SYS a0072777284, 7300-7306.

Leptobrachella tengchongensis $(\mathbf{n}=\mathbf{6})$ : China: Yunnan Province: Baoshan City: Mt. Gaoligong: SYS a004600 (holotype), 4596-4599, 4601-4602.

Leptobrachella wubuangmontis $(\mathbf{n}=12)$ : China: Guangxi Province: Pubei County: Mt. Wuhuang: SYS a003500/CIB107274, SYS a000578, 0580-0581, 34853489, 3499, 3504-3506.

Leptobrachella yunkaiensis ( $\mathbf{n}=\mathbf{8})$ : China: Guangdong Province: Maoming City: Dawuling Forest Station: SYS a004664/CIB107272, SYS a004663, 4665-4669, 4690.

\section{Supplementary material I}

Table S1. Pairwise distances based on $16 \mathrm{~S}$ gene among all sample used in this study Authors: Jian Wang, Zhi-Tong Lyu, Shuo Qi, Zhao-Chi Zeng, Wen-Xiang Zhang, Long-Shan Lu, Ying-Yong Wang

Data type: phylogenetic

Explanation note: Genetic distances among all Leptobrachella samples.

Copyright notice: This dataset is made available under the Open Database License (http://opendatacommons.org/licenses/odbl/1.0/). The Open Database License $(\mathrm{ODbL})$ is a license agreement intended to allow users to freely share, modify, and use this Dataset while maintaining this same freedom for others, provided that the original source and author(s) are credited.

Link: https://doi.org/10.3897/zookeys.955.55939.suppl1 